ARTICLE

\title{
Theranostic near-infrared-Ilb emitting nanoprobes for promoting immunogenic radiotherapy and abscopal effects against cancer metastasis
}

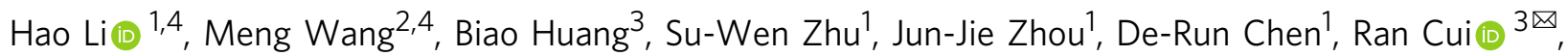
Mingxi Zhang (i) ${ }^{2 凶} \&$ Zhi-Jun Sun (10) ${ }^{1 凶}$

Radiotherapy is an important therapeutic strategy for cancer treatment through direct damage to cancer cells and augmentation of antitumor immune responses. However, the efficacy of radiotherapy is limited by hypoxia-mediated radioresistance and immunosuppression in tumor microenvironment. Here, we construct a stabilized theranostic nanoprobe based on quantum dots emitting in the near-infrared IIb (NIR-IIb, 1,500-1,700 nm) window modified by catalase, arginine-glycine-aspartate peptides and poly(ethylene glycol). We demonstrate that the nanoprobes effectively aggregate in the tumor site to locate the tumor region, thereby realizing precision radiotherapy with few side-effects. In addition, nanoprobes relieve intratumoral hypoxia and reduce the tumor infiltration of immunosuppressive cells. Moreover, the nanoprobes promote the immunogenic cell death of cancer cells to trigger the activation of dendritic cells and enhance T cell-mediated antitumor immunity to inhibit tumor metastasis. Collectively, the nanoprobe-mediated immunogenic radiotherapy can boost the abscopal effect to inhibit tumor metastasis and prolong survival.

\footnotetext{
${ }^{1}$ The State Key Laboratory Breeding Base of Basic Science of Stomatology (Hubei-MOST) \& Key Laboratory of Oral Biomedicine Ministry of Education, School \& Hospital of Stomatology, Wuhan University, 430079 Wuhan, China. ${ }^{2}$ State Key Laboratory of Advanced Technology for Materials Synthesis and Processing, Wuhan University of Technology, 430070 Wuhan, China. ${ }^{3}$ College of Chemistry and Molecular Sciences, Wuhan University, 430072 Wuhan, China. ${ }^{4}$ These authors contributed equally: Hao Li, Meng Wang. ${ }^{凶}$ email: cuiran@whu.edu.cn; mxzhang@whut.edu.cn; sunzj@whu.edu.cn
} 
C ancer, as a major public health problem, has been predicted in recent years to become the leading cause of death worldwide $^{1}$. Radiotherapy (RT) is an important therapeutic strategy for cancer treatment and is applied in $\sim 50 \%$ of patients who require curative-intent treatment for solid tumors ${ }^{2}$. However, some intrinsic shortcomings of RT still limit its clinical applications. When the radiation beam is applied locally to the tumor site, neighboring healthy tissues are also exposed to radiation, leading to normal tissue toxicity ${ }^{3}$. Therefore, accurate delineation of target tumor is prerequisite for precision RT. In addition, cancer cells usually resist X-ray radiation because of hypoxic conditions in the tumor microenvironment (TME), a common characteristic feature of most solid tumors ${ }^{4,5}$. Moreover, $\mathrm{RT}$ is generally prescribed for the treatment of localized tumors but ignores the evidence that the complex microenvironmental changes triggered by RT can also increase metastatic risks and counteract the long-term efficacy of the treatment ${ }^{6,7}$.

Notably, RT not only exerts direct cytotoxic effects on cancer cells through DNA damage by ionizing radiation and oxidative damage but also augments immune responses against tumors which have been proven essential for radiation-induced tumor regression ${ }^{8}$. One common hypothesis is that localized RT initiates immunogenic cell death (ICD) of cancer cells and then induces presentation by dendritic cells (DCs), leading to prime antitumor immunity mediated by tumor-specific cytotoxic T cells $s^{9,10}$. More importantly, localized RT not only enriches the cytotoxic $\mathrm{T}$ cell infiltrate in tumor tissue resulting in regression of the primary tumor but also produces systemic tumor-directed immunity, leading to elimination of metastatic cancer in the non-irradiated field (called the abscopal effect) and long-term immunological memory, especially when combined with immunotherapy ${ }^{11,12}$. Previous studies indicated that the combination strategy of RT and immunotherapy via immune checkpoint blockade (ICB) provides an opportunity to boost abscopal response rates ${ }^{11}$. Unfortunately, even when combining RT with immunotherapy, the RT-induced antitumor immune response and abscopal effect are stifled by immunosuppression or tolerance in the TME ${ }^{8,11}$. Hypoxia is an important obstacle contributing to immunosuppression and facilitating tumor recurrence, metastasis, and resistance to $\mathrm{RT}^{13,14}$. Hypoxia-inducible factor $1 \alpha$ (HIF-1 $\alpha$ ) subsequently stimulated by hypoxia plays a central role in hypoxia-induced resistance to radiation treatment ${ }^{13,15}$. Hypoxic cancer cells can be resistant to RT directly by HIF-1 $\alpha$ signaling ${ }^{15}$. Moreover, HIF-1 $\alpha$ signaling can lead to damage to antigen presentation and resistance of cancer cells to $\mathrm{T}$ cell-mediated cytotoxicity ${ }^{16}$. In addition to activation of HIF-1a signaling, hypoxia in tumors can also attract immunosuppressive cells, such as regulatory T cells (Tregs) and M2-like macrophages, into the TME. M2-like macrophages generally demonstrate immunosuppression and promotion of cancer growth by suppressing $\mathrm{T}$ cell function ${ }^{17-19}$. Thus, relieving the hypoxic environment in the TME can effectively improve the RT-induced antitumor immune response and abscopal effect.

With the rapid development of nanotechnology, applications of nanoparticles to overcome resistance to RT and enhance radiation efficacy against tumors have been realized in many preclinical studies ${ }^{20,21}$. Several nanoparticles with high atomic number (high- $Z$ ) elements can act as radiosensitizers and promote radiation energy deposition to enhance RT-induced direct damage to cancer cells ${ }^{22,23}$. Nanoparticles were also used to increase the radiosensitivity of cancer cells and weaken the immunosuppressive effect in the TME by relieving intratumoral hypoxia through peroxide hydrogen $\left(\mathrm{H}_{2} \mathrm{O}_{2}\right)$ decomposition and $\mathrm{O}_{2}$ production ${ }^{24-27}$. However, it is very difficult to trace the biodistribution of these nanoagents in living bodies, which may cause difficulty in determining the scope and time window of RT. Quantum dots (QDs) emitting at the long end of the second nearinfrared window (NIR-IIb, 1,500-1,700 nm) take advantage of deep tissue imaging with high spatial and temporal resolution, and can be used in combination with tumor imaging and treatment ${ }^{28,29}$. Moreover, the NIR-IIb emitting QDs also contain high $-\mathrm{Z}$ elements $(\mathrm{Pb}, \mathrm{Ag})$, which have the potential ability for radiosensitizing. Therefore, based on QD nanotechnology, we can develop a multifunctional nanoprobe to resolve the challenge of enhancing the precision and efficacy of RT.

In this study, we rationally design a stabilized theranostic nanoprobe based on NIR-IIb-emitting QDs, which can precisely image tumor regions with high resolution, promote the radiosensitivity and immunogenicity of cancer cells, and relieve intratumoral hypoxia to enhance RT-based therapy strategies. NIR-IIb-emitting PbS/CdS QDs are modified with catalase (Cat), arginine-glycine-aspartate (RGD) peptides and poly(ethylene glycol) (PEG) (Fig. 1a) ${ }^{30,31}$. The resulting QD-Cat-RGD nanoprobes can effectively aggregate in the tumor site, led by the RGD peptide, and emit in the NIR-IIb window to ascertain the shape and position of the tumor and determine the optimal time for RT. Moreover, the QD-Cat-RGD nanoprobes show a beneficial performance as a radiosensitizer with a high- $\mathrm{Z}$ atom $(\mathrm{Pb})$ in the nanoprobe, which not only promotes the direct killing effect of cancer cells but also enhances the immunogenicity of cancer cells via ICD to trigger a robust antitumor immune response. Cat, an efficient enzyme that promotes the rapid decomposition of $\mathrm{H}_{2} \mathrm{O}_{2}$, is modified on QDs to relieve intratumoral hypoxia enhancing the radiosensitization of cancer cells and reduce the infiltration of immunosuppressive cells to destroy the immunosuppression in the TME. This multifunctional nanoprobe-based immunogenic RT, combined with immunotherapy using anti-programmed cell death 1 antibodies (aPD-1), can efficiently promote RT-induced abscopal effects to inhibit tumor metastasis and prolong survival.

\section{Results}

Preparation and characterization of QD-Cat-RGD nanoprobes. NIR-IIb $\mathrm{PbS} / \mathrm{CdS}$ core-shell QDs emitting at $1600 \mathrm{~nm}$ were synthesized as previously described ${ }^{30,31}$. Oleyamine-branched poly(acrylic acid) (OPA) was coated on the surface of QDs to transfer them into aqueous solution. Then, 8-Arm PEG- $\mathrm{NH}_{2}$ molecules, RGD peptides, and Cat were conjugated on the surface of OPA-modified QDs via N-(3-(dimethylamino)propyl)- $\mathrm{N}^{\prime}$ ethylcarbodiimide hydrochloride (EDC) chemistry ${ }^{32}$. The carboxylic groups on QDs were activated by EDC molecules and then reacted with the amine groups on Cat to form amide bonds. Transmission electron microscopy (TEM) images demonstrated the regular granular structure and good dispersity of the QD-CatRGD nanoprobes (Fig. 1b). Dynamic light scattering (DLS) further revealed that the average hydrodynamic size of QD-CatRGD in aqueous solution was $46.7 \pm 1.1 \mathrm{~nm}$ (Supplementary Fig. 1a). In addition, the zeta potential of QD-Cat-RGD changed from -51.33 to $-1.12 \mathrm{mV}$ after conjugation (Supplementary Fig. 1b). The absorption spectrum and fluorescence emission spectrum of QD-Cat-RGD nanoprobes are shown in Supplementary Fig. 1c, d. The fluorescence quantum yield (QY) of the nanoprobe was calculated to be $2.2 \%$ (referenced to IR-26 dye with a QY of $0.05 \%)$.

To reveal the loading capacity of RGD and Cat on the QD-CatRGD nanoprobes, we used a standard BCA protein assay to quantify the unbound Cat after the conjugation and purification of nanoprobes and consequently obtained the amounts of Cat grafted on QDs. The molar ratio of grafted Cat onto QDs was approximately 2.5:1. We further used UV spectrometry to detect the absorbance at $220 \mathrm{~nm}$ to quantify the unbound RGD and obtain the amounts of RGD grafted on $\mathrm{QDs}^{33}$. We calculated that the molar ratio of grafted RGD to QDs was approximately 30:1. 
a

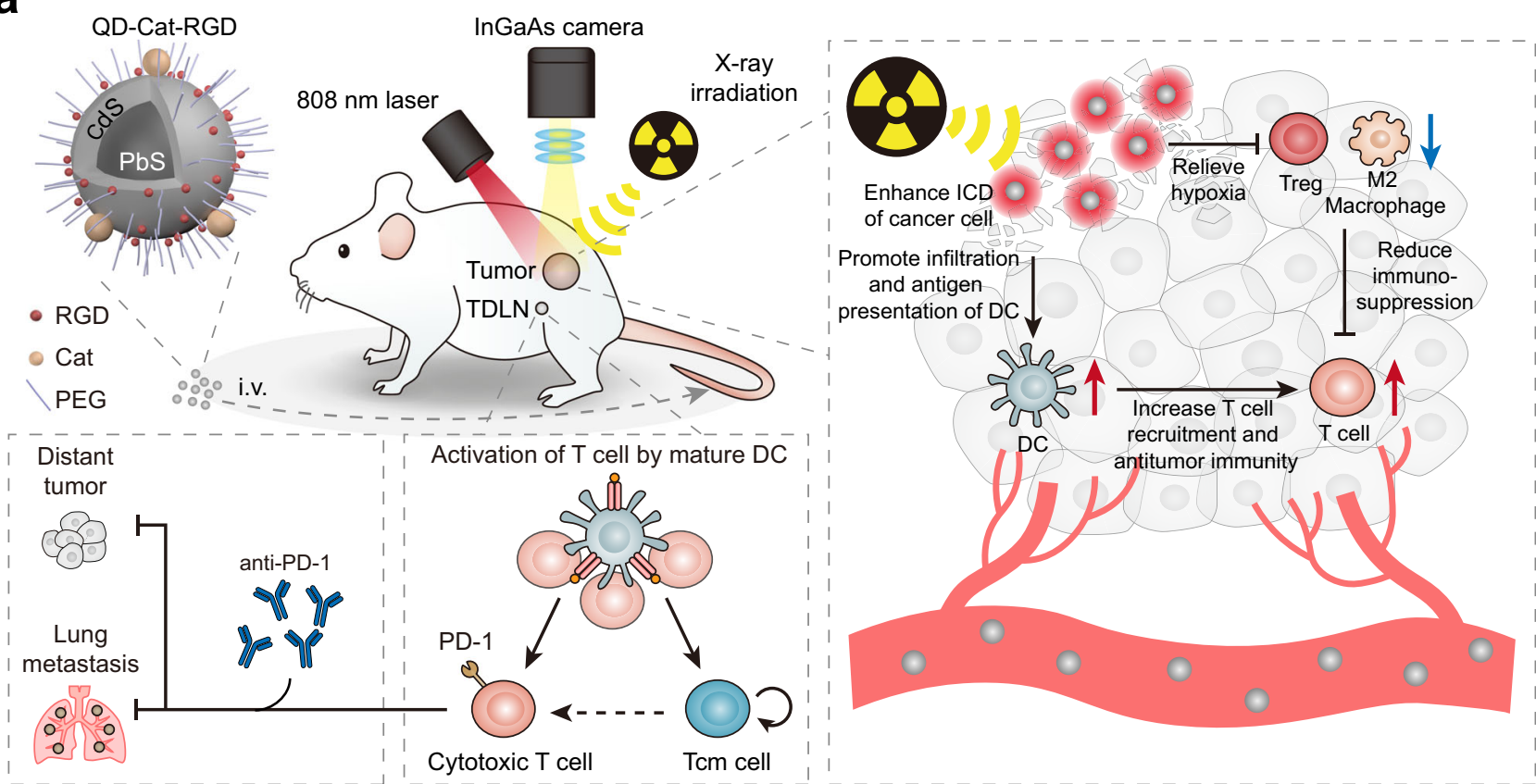

b

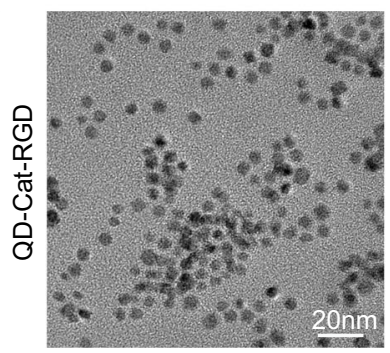

C

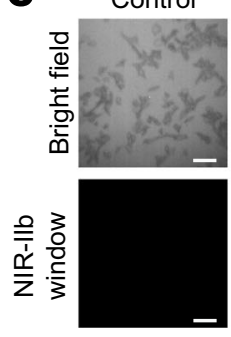

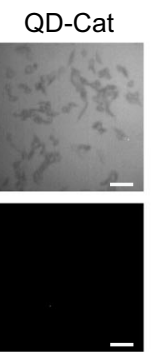
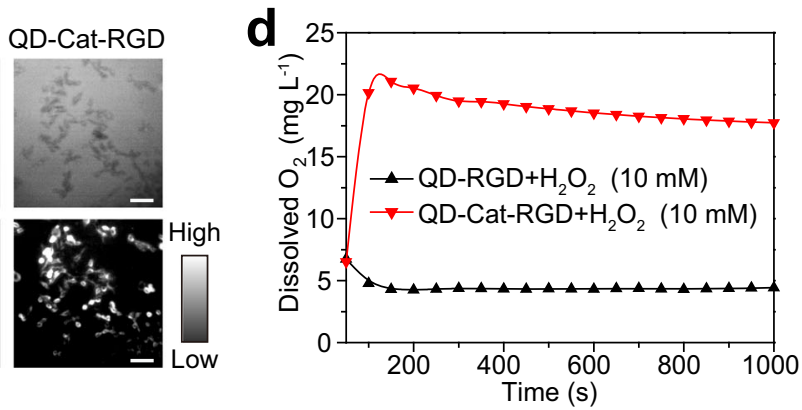

Fig. 1 Schematic and characterization of QD-Cat-RGD. a Schematic showing the mechanism by which QD-Cat-RGD nanoprobe-based RT boosts the antitumor immune response and abscopal effect to inhibit cancer metastases. b A TEM image of QD-Cat-RGD nanoprobes. Scale bar $=20 \mathrm{~nm}$. $\mathbf{c}$ Images of $4 T 1$ cells after incubation with PBS (control), QD-Cat (without RGD modification), or QD-Cat-RGD (with RGD modification) in the NIR-Ilb window and bright field. Scale bars $=50 \mu \mathrm{m}$. d Dissolved oxygen generation in $\mathrm{H}_{2} \mathrm{O}_{2}$ solutions (10 mM) with QD-RGD (without Cat modification) or QD-Cat-RGD. The TEM of the QD-Cat-RGD nanoprobe (b), the cell imaging experiments (c), and $\mathrm{H}_{2} \mathrm{O}_{2}$ decomposition experiment (d) were representative of those generated from three independent experiments. RGD arginine-glycine-aspartate peptides, Cat catalase, PEG poly(ethylene glycol), ICD immunogenic cell death, Treg regulatory T cell, DC dendritic cell, Tcm central memory T cell, i.v. intravenous, PD-1 programmed cell death 1, TDLN tumor-draining lymph node, NIR-IIb near-infrared IIb. Source data are provided as a Source Data file.

We next tested the stability of QD-Cat-RGD nanoprobes. The fluorescence intensity of QD-Cat-RGD nanoprobes dispersed in fetal bovine serum (FBS) was reduced by less than $3 \%$ after continuous $808 \mathrm{~nm}$ laser exposure for $1 \mathrm{~h}$ (Supplementary Fig. 1e), which confirmed the good fluorescence stability of the QD-Cat-RGD nanoprobe. In addition, we intravenously injected QD-Cat-RGD nanoprobes into $\mathrm{BALB} / \mathrm{c}$ mice and collected the feces for several days. As confirmed by the TEM image (Supplementary Fig. 2a), we found that QD-Cat-RGD nanoprobes remained intact even after being excreted from the body. Energy dispersive X-ray spectroscopy (EDS) analysis revealed nanoparticles extracted from the feces containing $\mathrm{Pb}$, S, and Cd species (Supplementary Fig. 2b), which demonstrated the excellent in vivo stability of QD-Cat-RGD nanoprobes.

The RGD peptides can be recognized by integrin $\alpha_{v} \beta_{3}$, which is overexpressed in the tumor-associated vasculature and several types of cancer cells, such as $4 \mathrm{~T} 1$ murine breast cancer cells and B16F10 murine melanoma cells ${ }^{34,35}$. With RGD modification, QD-Cat-RGD nanoprobes acquired the ability to target $4 \mathrm{~T} 1$ cells (integrin $\alpha_{v} \beta_{3}$-positive) compared with the QDs without RGD modification (Fig. 1c). $\mathrm{H}_{2} \mathrm{O}_{2}$ is produced by malignant cells and excessively accumulates in tumor tissue ${ }^{20,36}$. Cat coated on the surface of the QDs was used to produce $\mathrm{O}_{2}$ and relieve hypoxia by catalytic decomposition of $\mathrm{H}_{2} \mathrm{O}_{2}$ in tumors. As measured by the oxygen probe, rapid generation of $\mathrm{O}_{2}$ could be observed in $\mathrm{H}_{2} \mathrm{O}_{2}$ solution $(10 \mathrm{mM})$ in the presence of QD-Cat-RGD (Fig. 1d). The above results demonstrated that the QD-Cat-RGD nanoprobes integrated multiple functions of imaging guidance, tumor targeting, and $\mathrm{O}_{2}$ production.

Imaging in the NIR-IIb window with high clarity. The bright $1,600 \mathrm{~nm}$ fluorescence of $\mathrm{PdS} / \mathrm{CdS} \mathrm{QDs}$ is ideal for imaging in vivo because of its high spatial and temporal resolution ${ }^{30}$. To evaluate the image-guided properties of QD-Cat-RGD nanoprobes in vivo, $4 \mathrm{~T} 1$ tumor-bearing $\mathrm{BALB} / \mathrm{c}$ mice were intravenously injected with QD-Cat-RGD $\left(150 \mu \mathrm{L}, 2 \mathrm{mg} \mathrm{mL}^{-1}\right)$ or QDCat $\left(150 \mu \mathrm{L}, 2 \mathrm{mg} \mathrm{mL}^{-1}\right)$ nanoprobes. Then, a wide-field 2D InGaAs camera was used to collect emission photons between 
a
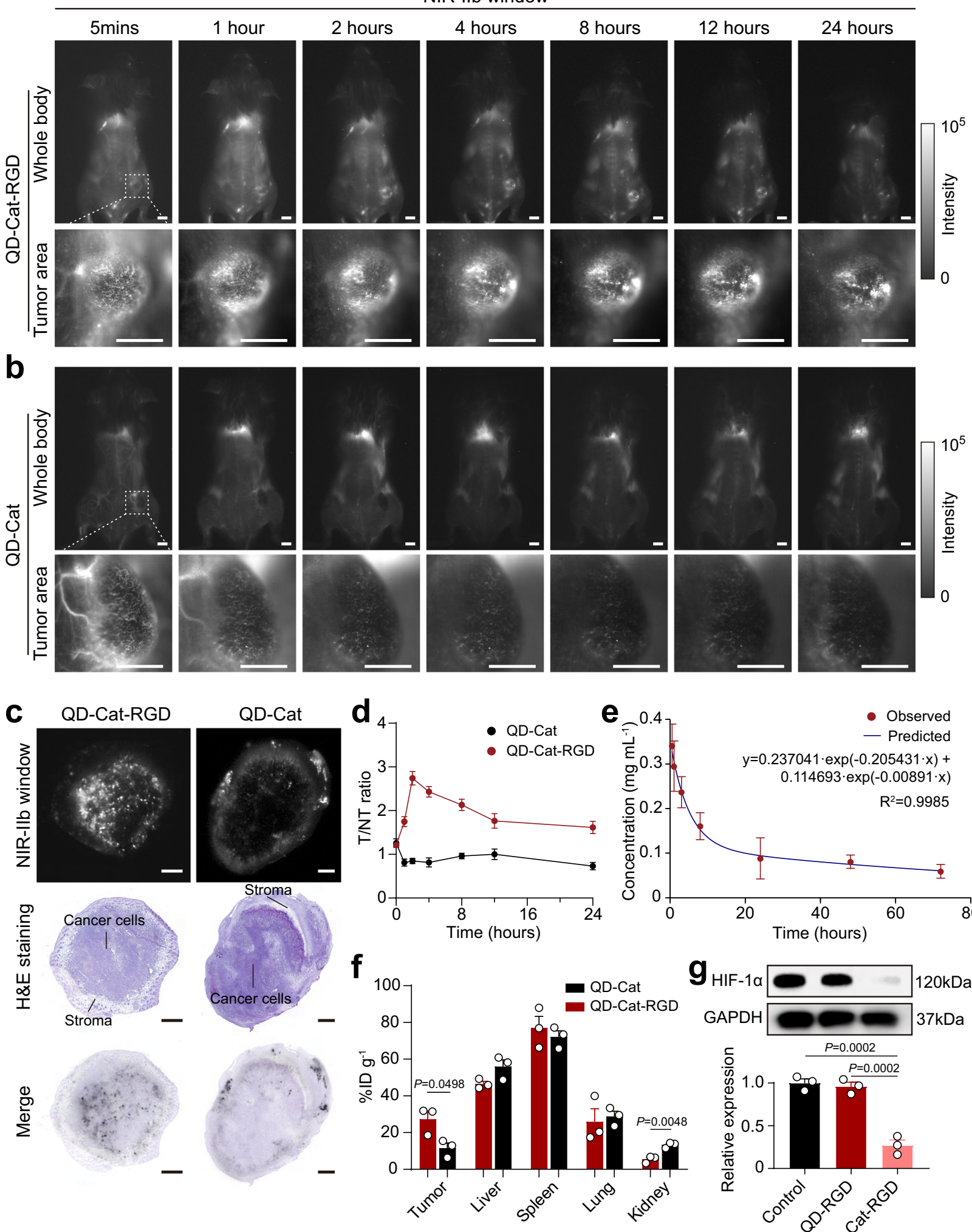

- Observed

- Predicted

$y=0.237041 \cdot \exp (-0.205431 \cdot x)+$

$0.114693 \cdot \exp (-0.00891 \cdot x)$

$\mathrm{R}^{2}=0.9985$
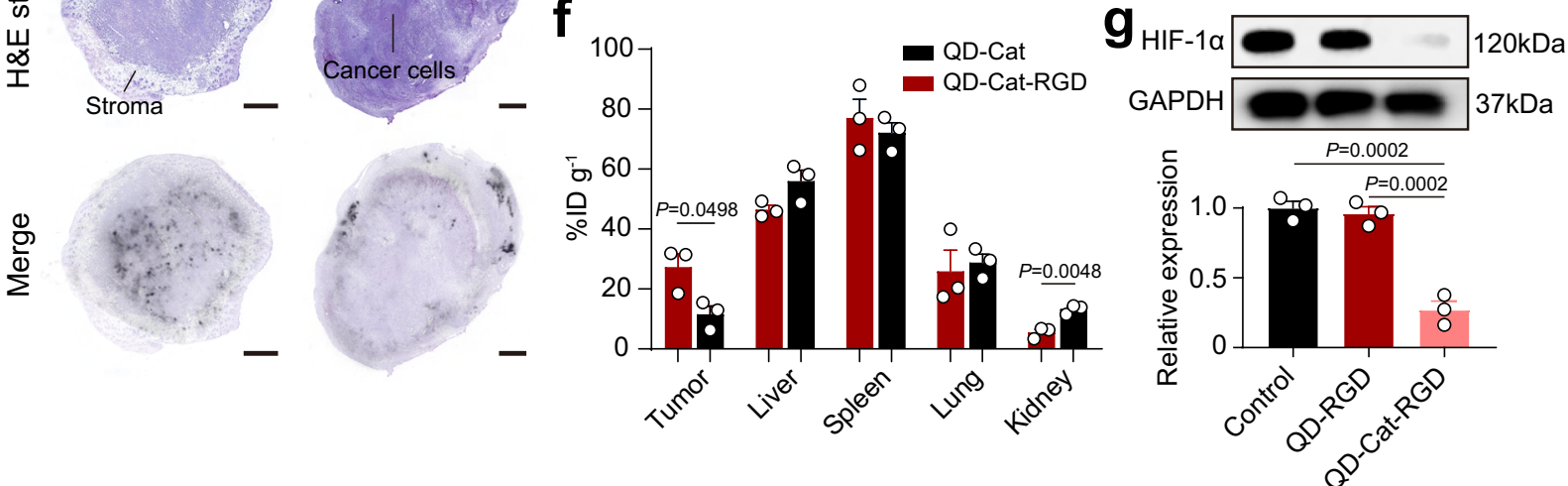

1,500 and $1,700 \mathrm{~nm}$ under $808 \mathrm{~nm}$ laser excitation at a power density of $25 \mathrm{~mW} \mathrm{~cm}^{-2}$. After QD-Cat-RGD injection, the vasculature system of the mouse body was first imaged in the NIRIIb window including the abnormal blood vasculature in the tumor (Fig. 2a). Due to the suppressed photon scattering and diminished autofluorescence in the NIR-IIb window, we could monitor the change in the internal tumor region over time with high-resolution fluorescence imaging precise profiling (Fig. 2a). The strong signals focused at the tumor site confirmed the gradual infiltration of QD-Cat-RGD nanoprobes with an excellent tumor-targeting ability (Fig. 2a). However, the QD-Cat nanoprobes without RGD modification did not show this ability, with 
Fig. 2 In vivo fluorescence imaging in the NIR-Ilb window and biodistribution of QD-Cat-RGD. $\mathbf{a}, \mathbf{b}$ Whole-body and high-magnification fluorescent images of $4 \mathrm{~T} 1$ tumor-bearing mice intravenously injected with QD-Cat-RGD (a) or QD-Cat (b) nanoprobes at different time points (5 min, $1 \mathrm{~h}, 2 \mathrm{~h}, 4 \mathrm{~h}, 8 \mathrm{~h}$, $12 \mathrm{~h}, 24 \mathrm{~h}$ ) in the NIR-IIb window. Scale bars $=5 \mathrm{~mm}$. The excitation power density was $50 \mathrm{~mW} \mathrm{~cm}{ }^{-2}$ provided by an $808 \mathrm{~nm}$ laser. c Serial sections of tumor tissue imaged in the NIR-Ilb window or stained with H\&E. Scale bars $=1 \mathrm{~mm}$. The excitation power density for NIR-IIb fluorescence imaging was $25 \mathrm{~mW} \mathrm{~cm}^{-2}$ provided by an $808 \mathrm{~nm}$ laser. The fluorescent images in the NIR-Ilb window (a-c) were representative of those generated from three mice each group. d The T (tumor)/NT (nontumor) ratio of QD-Cat-RGD or QD-Cat at different time points ( 5 min, $1 \mathrm{~h}, 2 \mathrm{~h}, 4 \mathrm{~h}, 8 \mathrm{~h}, 12 \mathrm{~h}, 24 \mathrm{~h}$ ). e The blood concentration of $\mathrm{Pb}$ in $\mathrm{BALB} / \mathrm{c}$ mice at different time points $(0.5,1,3,8,24,48,72 \mathrm{~h})$ after intravenous injection with QD-Cat-RGD nanoprobes. $\mathbf{f}$ Delivery efficiency for injected dose (ID) of QD-Cat-RGD or QD-Cat in tumors and main organs (livers, spleens, lungs, and kidneys) of mice injected with QD-CatRGD or QD-Cat at $24 \mathrm{~h}$ p.i. $\mathbf{g}$ The protein expression level of HIF-1 $\alpha$ in $4 \mathrm{~T} 1$ tumor tissue in mice at $24 \mathrm{~h}$ after intravenous injection with PBS (control), QDRGD $\left(150 \mu \mathrm{L}, 2 \mathrm{mg} \mathrm{mL}^{-1}\right)$, and QD-Cat-RGD $\left(150 \mu \mathrm{L}, 2 \mathrm{mg} \mathrm{mL}^{-1}\right)$ measured by western blot analysis. Western blot was done thrice. All data are shown as the mean \pm s.e.m. of $(n=3)$ and $n$ represents the number of independent samples. Statistical significance was calculated via two-tailed Student's $t$-test (f) and one-way ANOVA with Tukey's multiple comparisons test (g). NIR-Ilb near-infrared IIb. Source data are provided as a Source Data file.

weak signals found in tumor tissue (Fig. 2b). The tumor-tonormal tissue (T/NT) signal ratio of QD-Cat-RGD increased sharply and peaked at approximately $2 \mathrm{~h}$ postinjection (p.i.) with a T/NT ratio of approximately 2.742 , which was higher than the $\mathrm{T} / \mathrm{NT}$ ratio of QD-Cat (Fig. 2d). These results can help to determine the optimum time for RT when the QD-Cat-RGD nanoprobes accumulate maximally in tumors compared with normal tissues to improve the efficiency of radiosensitizers. In addition to tumors, QD-Cat-RGD nanoprobes also accumulated in the liver, spleen, and lung $24 \mathrm{~h}$ p.i., with little retention in other organs including the heart and kidney (Supplementary Fig. 2c).

To explore the precise position of QD-Cat-RGD infiltrated in tumor tissue, serial slices of tumor tissue were imaged in the NIRIIb window and stained with hematoxylin and eosin (H\&E). The merged images showed that the QD-Cat-RGD nanoprobes with RGD modification could deeply infiltrate in the tumor tissue (Fig. 2c). Without RGD modification, QD-Cat nanoprobes were mainly found in the stroma which was infiltrated with supporting vasculatures (Fig. 2c). Moreover, we sorted the live CD45- cells which were mainly cancer cells from tumor tissue by fluorescentactivated cell sorting (FACS; the sorting gating strategy is shown in Supplementary Fig. 3a), and investigated the content of nanoprobe associated element $\mathrm{Pb}$ in these cells by inductively coupled plasma optical emission spectrometry (ICP-OES). The results showed that the content of $\mathrm{Pb}$ in the QD-Cat-RGD group were higher than that in QD-Cat group (Supplementary Fig. 3b), which suggested that the RGD modification significantly improved the targeting efficiency of nanoprobes to cancer cells in vivo. To further reveal the relationship between location of QD-Cat-RGD nanoprobes and hypoxic region in tumor, serial slices of tumor tissue from QD-Cat-RGD group were imaged in the NIR-IIb window, stained with H\&E, and immunostained with HIF-1 $\alpha$ by immunohistochemistry (IHC). As shown in Supplementary Fig. 4, the fluorescent signals in NIR-IIb can be found in the Region 1 in the tumor (Supplementary Fig. 4d), which was near the stroma infiltrated with several vasculatures (Supplementary Fig. 4e) and with weak nuclear HIF-1a immunostaining (Supplementary Fig. 4g). Moreover, the fluorescent signals in NIR-IIb can also be found in the Region 2 in the tumor (Supplementary Fig. 4d), which was away from vasculatures (Supplementary Fig. 4f) and with strong nuclear HIF-1a immunostaining (Supplementary Fig. 4h). These results suggested that QD-Cat-RGD nanoprobes with the targeting property of RGD might diffuse in the tumor tissue.

Here, we selected $\mathrm{Pb}$ element measured by ICP-OES to investigate the pharmacokinetics of nanoprobes and biodistributions in the main organs of the body, including the liver, spleen, lungs, and kidneys, as well as tumors after blood circulation. The blood circulation half-time $\left(T_{1 / 2}\right)$ of QD-Cat-RGD was calculated to be approximately $3.37 \mathrm{~h}$ (Fig. 2e) according to a twocompartment model ${ }^{37}$. The systemic distribution of $\mathrm{Pb}$ revealed a $17.67 \%$ injected dose (ID) $\mathrm{g}^{-1}$ tumor accumulation efficiency of QD-Cat-RGD (Fig. 2f), which was higher than the $6.84 \% \mathrm{ID} \mathrm{g}^{-1}$ tumor accumulation efficiency of QD-Cat (Fig. 2f). The above results demonstrated a good property of QD-Cat-RGD by comparing with the QD-Cat (without RGD) group.

We then tested the anti-hypoxia ability of QD-Cat-RGD nanoprobes in vivo. At $24 \mathrm{~h}$ after the injection of QD-Cat-RGD or QD-RGD (without Cat modification), the tumor tissues were harvested from the mice. The expression of HIF-1a in tumor tissue as an index of hypoxia was analyzed by western blot, which clearly showed that the expression level of HIF-1a was significantly reduced in the QD-Cat-RGD group compared with the QD-RGD group and control group (Fig. 2g). Together, this evidence confirmed that the well-designed nanoprobe could specifically target the tumor and relieve hypoxia in the TME for RT treatment.

Reinforcement of ICD of cancer cells induced by RT. Since QDCat-RGD based on $\mathrm{PbS} / \mathrm{CdS} \mathrm{QDs}$ contain high- $\mathrm{Z}$ element $\mathrm{Pb}$ and Cat which could relieve hypoxia via the catalysis of endogenous $\mathrm{H}_{2} \mathrm{O}_{2}$ in the TME, they have a strong potential to promote RT efficacy. To study the effect of nanoprobes and RT on cells under hypoxia, cells were incubated under hypoxic condition $\left(37^{\circ} \mathrm{C}, 1 \%\right.$ $\mathrm{O}_{2}, 5 \% \mathrm{CO}_{2}$ ). To evaluate the QD-Cat-RGD nanoprobe's potential as a radiosensitizer for RT, a colony formation assay was conducted to assess the sensitivity of $4 \mathrm{~T} 1$ cancer cells to different treatments in hypoxia in vitro. The results showed that the proliferative activation of $4 \mathrm{~T} 1$ cells treated with QD-RGD- or QDCat-RGD-based RT in hypoxia significantly decreased compared with RT in hypoxia (Fig. 3a, b). The radiosensitization of QDRGD and QD-Cat-RGD was further confirmed by $\mathrm{p}-\mathrm{H} 2 \mathrm{AX}$ staining, which reflected the DNA damage of cancer cells ${ }^{38,39}$. Consistent with the results of the colony formation assay, the fluorescence intensity of $\mathrm{p}-\mathrm{H} 2 \mathrm{AX}$ in the nuclei of $4 \mathrm{~T} 1$ cells was significantly increased in the hypoxia + RT + QD-RGD and hypoxia + RT + QD-Cat-RGD groups compared with the RT group under hypoxic conditions (Fig. 3c). In addition, an annexin $\mathrm{V} /$ propidium iodide (PI) staining assay suggested that the number of vital $4 \mathrm{~T} 1$ cells decreased in the hypoxia $+\mathrm{RT}+\mathrm{QD}-\mathrm{RGD}$ and hypoxia + RT + QD-Cat-RGD groups compared with the other groups (Fig. 3d). We also tested the radiosensitization of QD-Cat-RGD nanoprobes under normoxic conditions and found that the nanoprobes also had radiosensitizing effect under normoxic conditions (Supplementary Fig. 5).

Considering that QD-Cat-RGD nanoprobes significantly promoted the RT efficacy of directly killing cancer cells, we then evaluated the effect of QD-Cat-RGD-based RT on promoting the ICD of cancer cells. RT can trigger ICD of cancer cells through emission of tumor-associated antigen and ICD-associated danger-associated molecular patterns (DAMPs), such as exposure of calreticulin on the surface of dying cancer cells, and the release 
a

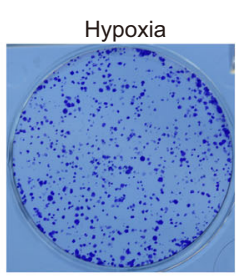

b

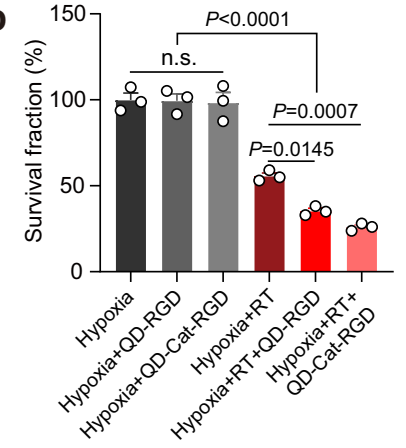

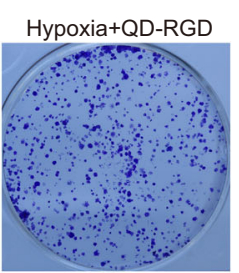

C Hypoxia

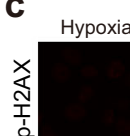

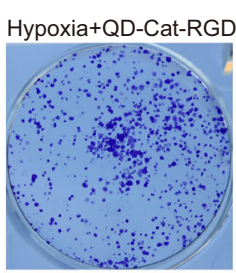

Hypoxia+ QD-RGD 2 Hypoxia+ QD-RGD QD-Cat-RGD
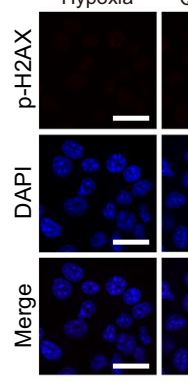

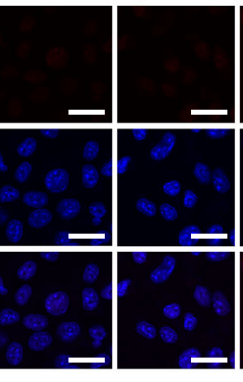

Hypoxia+ QD-Cat-RGD

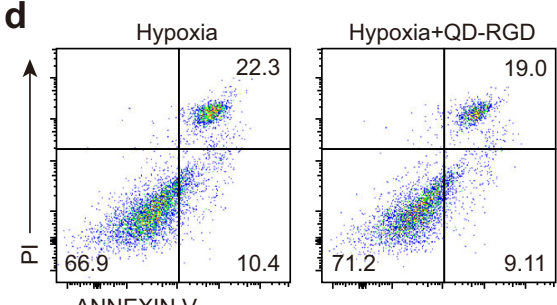

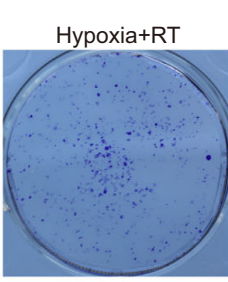

Hypoxia+
RT
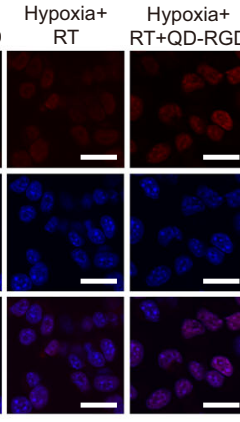

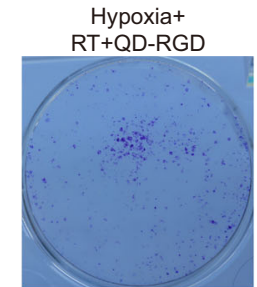

Hypoxia+ $R T+Q D-C a t-R G D$

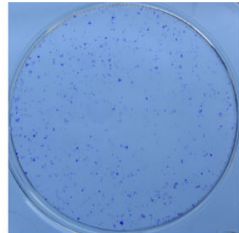

$P<0.0001$
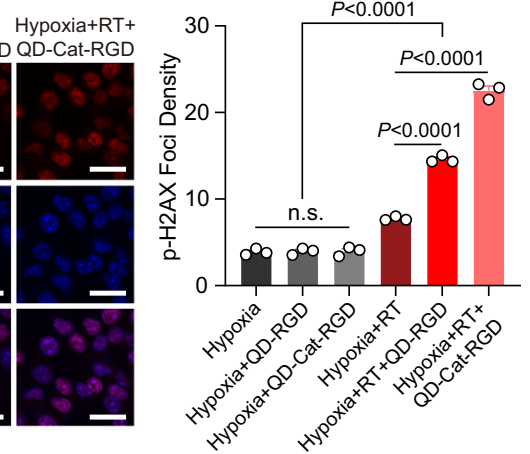

Hypoxia+

Hypoxia+
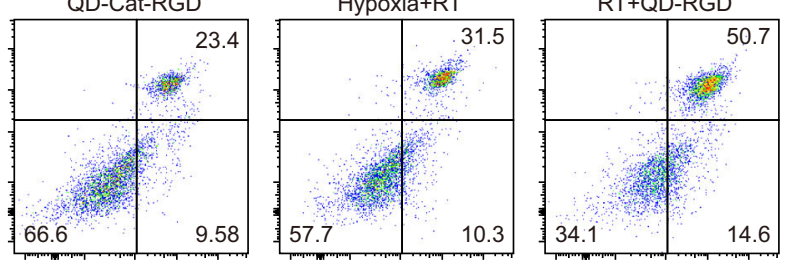

RT+QD-Cat-RGD

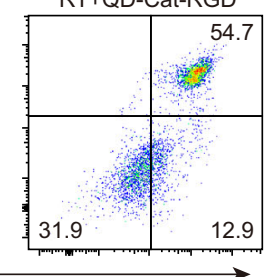

e
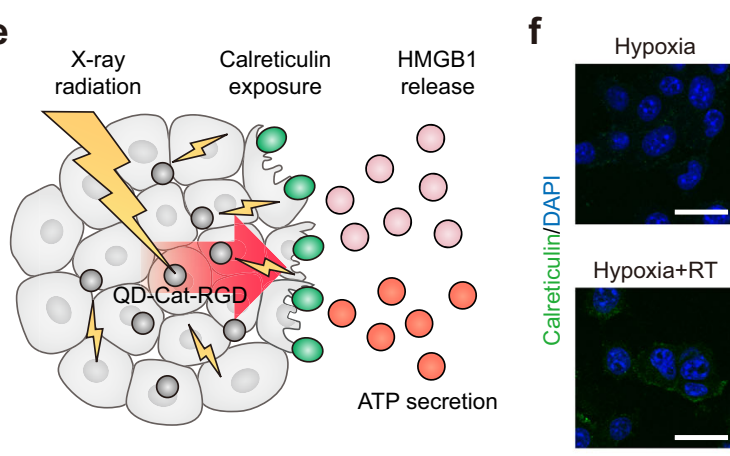

Hypoxia+

Hypoxia+

QD-Cat-RGD $\mathbf{g}$

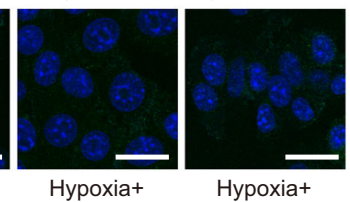

$\begin{array}{cc}\text { Hypoxia+ } & \text { Hypoxia+ } \\ \text { RT+QD-RGD } & \text { RT+QD-Cat-RGD }\end{array}$
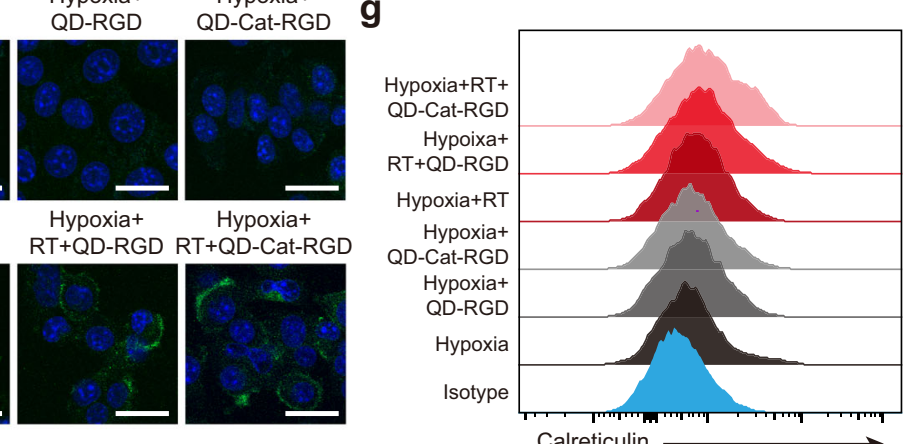

h

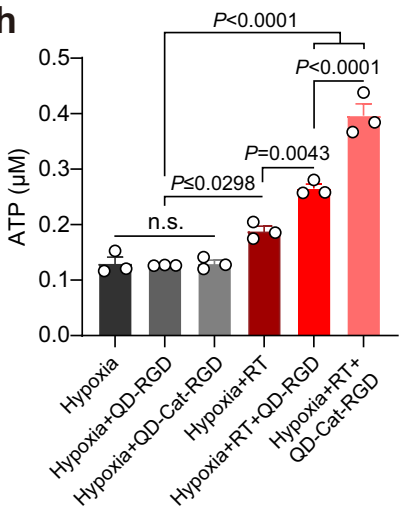

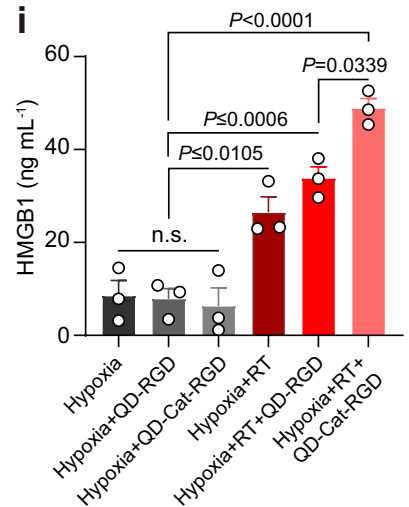

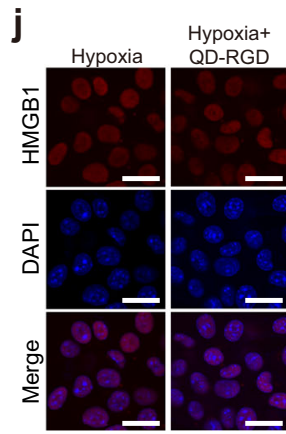

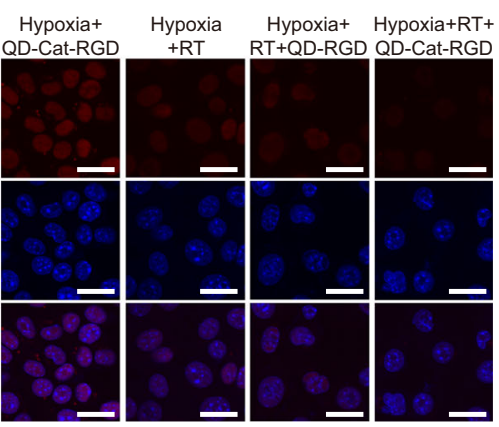

of large amounts of high-mobility group box 1 (HMGB1) and adenosine triphosphate (ATP) into the extracellular milieu, consequently resulting in activation of antitumor immunity (Fig. 3e $)^{9}$. As measured by fluorescence imaging and flow cytometry, the surface expression of calreticulin in $4 \mathrm{~T} 1$ cells in the hypoxia + RT + QD-RGD and hypoxia + RT + QD-Cat-
RGD groups was significantly increased compared with that in the $4 \mathrm{~T} 1$ cells treated with hypoxia + RT only (Fig. 3f, g and Supplementary Fig. 6). We also tested ATP and HMGB1 release in cancer cells, two crucial signals during DC recruitment and maturation ${ }^{9}$. At $24 \mathrm{~h}$ after RT, $4 \mathrm{~T} 1$ cells treated with QD-RGDor QD-Cat-RGD-based RT under hypoxic conditions exhibited 
Fig. 3 QD-Cat-RGD as a radiosensitizer for enhancing ICD of cancer cells under hypoxic conditions. 4T1 cells were incubated under hypoxic conditions $\left(37^{\circ} \mathrm{C}, 1 \% \mathrm{O}_{2}, 5 \% \mathrm{CO}_{2}\right.$ ) and irradiated with X-ray doses of $6 \mathrm{~Gy}$ in vitro. a Representative photographs of stained colonies of $4 \mathrm{T1}$ cells treated with hypoxia, hypoxia + QD-RGD, hypoxia + QD-Cat-RGD, hypoxia + RT (6 Gy), hypoxia + RT + QD-RGD (6 Gy) or hypoxia + RT + QD-Cat-RGD (6 Gy) after 7 days. The photographs of stained colonies (a) were representative of those generated from three independent samples each group. $\mathbf{b}$ Histogram plot of the survival fraction of $4 \mathrm{~T} 1$ cells with different treatments. c Immunofluorescence staining of p-H2AX (Ser139) and quantitative analysis of foci density of foci per cell at $4 \mathrm{~h}$ after different treatments. Scale bars $=25 \mu \mathrm{m}$. d Apoptosis analysis measured by flow cytometry of $4 \mathrm{~T} 1 \mathrm{cells}$ at $24 \mathrm{~h}$ after different

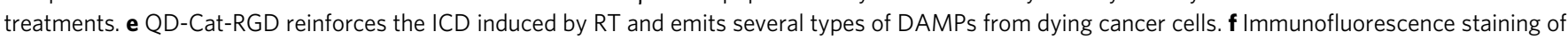
calreticulin of $4 \mathrm{~T} 1$ cells with different treatments after $24 \mathrm{~h}$. Scale bars $=25 \mu \mathrm{m}$. $\mathbf{g}$ Flow cytometry analysis of surface calreticulin expression in $4 \mathrm{~T} 1 \mathrm{cells}$ with different treatments after $24 \mathrm{~h}$. $\mathbf{h}$ Histogram plot of secreted ATP production by $4 \mathrm{T1}$ cells with different treatments after $24 \mathrm{~h}$. $\mathbf{i}$ Histogram plot of HMGB1 released by $4 \mathrm{~T} 1$ cells with different treatments after $24 \mathrm{~h}$. $\mathbf{j}$ Immunofluorescence staining of HMGB1 in $4 \mathrm{T1}$ cells with different treatments after $24 \mathrm{~h}$. Scale bars $=25 \mu \mathrm{m}$. The images of immunofluorescence staining $(\mathbf{c}, \mathbf{f}, \mathbf{j})$ were representative of those generated from three independent samples each group. All data are shown as the mean \pm s.e.m. of $(n=3)$ and $n$ represents the number of independent samples. Statistical significance was calculated via one-way ANOVA with Tukey's multiple comparisons test. n.s. not significant. Source data are provided as a Source Data file.

significantly increased ATP secretion (Fig. 3h) and HMGB1 release (Fig. 3i), as measured by ATP assay and enzyme-linked immunosorbent assay (ELISA). With HMGB1 release, the expression of HMGB1 in the cell nuclei of 4T1 cells in the hypoxia + $\mathrm{RT}+\mathrm{QD}-\mathrm{RGD}$ and hypoxia + RT + QD-Cat-RGD groups decreased compared with those only treated with hypoxia $+\mathrm{RT}$ (Fig. 3j).

Moreover, we further tested the effect of QD-Cat-RGD-based $\mathrm{RT}$ on promoting ICD in vivo. The $4 \mathrm{~T} 1$ tumor-bearing BALB/c mice were intravenously injected with a single dose of QD-CatRGD $\left(150 \mu \mathrm{L}, 2 \mathrm{mg} \mathrm{mL}^{-1}\right)$ or QD-RGD $\left(150 \mu \mathrm{L}, 2 \mathrm{mg} \mathrm{mL}^{-1}\right)$. Then, the mice were treated with localized RT ( $12 \mathrm{~Gy})$ at $2 \mathrm{~h}$ p.i. The tumor tissues were harvested at $24 \mathrm{~h}$ after the injection of QD-Cat-RGD or QD-RGD. The results showed that tumor tissue significantly increased the surface expression of calreticulin and ATP and HMGB1 concentrations (Supplementary Fig. 7a-c) at $24 \mathrm{~h}$ after the mice treated with QD-RGD- or QD-Cat-RGDbased RT, which was consistent with the in vitro results. This evidence demonstrated that QD-Cat-RGD containing high-Z element $\mathrm{Pb}$ and Cat could significantly promote $\mathrm{RT}$ efficiency and reinforce the ICD of cancer cells under hypoxic conditions, which could potentially promote more recruitment and maturation of $\mathrm{DCs}^{40}$

Relieving hypoxia to overcome immunosuppression in the TME. To examine the proposed immune activation of the combination of RT and the QD-Cat-RGD strategy, a mouse model of $4 \mathrm{~T} 1$ breast cancer was used. When the tumors reached approximately $100 \mathrm{~mm}^{3}$, 4T1 tumor-bearing $\mathrm{BALB} / \mathrm{c}$ mice were intravenously injected with a single dose of QD-Cat-RGD $(150 \mu \mathrm{L}$, $2 \mathrm{mg} \mathrm{mL}^{-1}$ ) or phosphate-buffered saline (PBS, $150 \mu \mathrm{L}$ ). Considering that NIR-IIb fluorescence imaging can show the area of tumors in mice after injection of QD-Cat-RGD, we first determined the location and range of RT with the help of NIR-IIb imaging (Supplementary Fig. 8a). Then, we used the lead shield to localize the range of RT determined by previous imaging (Supplementary Fig. 8b, c). The mice were treated with RT (12 Gy) at $2 \mathrm{~h}$ p.i. After 14 days, the lymphocytes in tumor tissues and tumor-draining lymph nodes (TDLNs) were analyzed by flow cytometry to demonstrate the immune-related function of QDCat-RGD in vivo (Fig. 4a). The tumor volume and weight results showed that tumors in the RT and RT + QD-Cat-RGD groups were significantly reduced compared with those in the control and QD-Cat-RGD groups (Fig. 4b, c and Supplementary Fig. 10). Compared with the RT group, the RT + QD-Cat-RGD group showed a stronger ability to inhibit tumor growth (Fig. $4 \mathrm{~b}, \mathrm{c}$ ). We also monitored the weight changes of mice after treatment. After 14 days, the body weights of the mice in the different groups were no significantly difference (Fig. 4 d).
We then tested the capacity of QD-Cat-RGD to relieve hypoxia in the TME. Hypoxia in the tumor site can lead to the recruitment of large numbers of macrophages and subsequently impact macrophage polarization by inducing macrophage differentiation towards an immunosuppressive M2-like phenotype (labeled by CD206) instead of an immunostimulatory M1like phenotype (labeled by CD80) in the $\mathrm{TME}^{17}$. In our study, compared with the RT group, the RT + QD-Cat-RGD group showed a stronger ability to inhibit M2-like macrophages $\left(\mathrm{CD} 206^{\text {high }} \mathrm{CD} 11 \mathrm{~b}^{+} \mathrm{F} 4 / 80^{+}\right.$) (Fig. 4e). Moreover, although there was no significant difference in the proportion of M1-like macrophages $\left(\mathrm{CD} 80^{\text {high }} \mathrm{CD} 11 \mathrm{~b}^{+} \mathrm{F} 4 / 80^{+}\right)$among these four groups (Supplementary Fig. 11a), the ratio of M1-like macrophages to M2-like macrophages was significantly increased in the RT + QD-Cat-RGD group compared with other the three groups (Fig. 4e). The presence of Tregs is also an important component for maintaining the hypoxia-induced immunosuppressive microenvironment ${ }^{41}$. We then tested Treg flow cytometry. Compared with the control group, Tregs $\left(\mathrm{CD} 4{ }^{+} \mathrm{CD} 25^{+}\right.$Foxp $\left.3^{+}\right)$ in the RT + QD-Cat-RGD group were more significantly reduced than those in the RT group (Fig. 4f and Supplementary Fig. 11c). The expression of HIF-1a may serve as a marker to reflect hypoxic conditions ${ }^{15}$. In the QD-Cat-RGD and RT + QD-CatRGD groups, reduced expression of HIF-1a in cell nuclei was observed in tumor tissues (Fig. 4g). This evidence showed that QD-Cat-RGD-based RT had a good ability to inhibit the growth of tumors and relieve intratumoral hypoxia to overcome immunosuppression in the TME.

Elicitation of $\mathbf{T}$ cell-mediated antitumor immunity. DCs are specialized antigen-presenting cells with essential roles in the generation of cancer-specific immunity triggered by different treatments ${ }^{42}$. ICD-driven DAMPs can attract DC recruitment and induce presentation of tumor-associated antigens by major histocompatibility complex (MHC)-II expression on DCs ${ }^{9}$. In addition, mature DCs express co-stimulatory molecules (CD80 and CD86) to induce the activation of T cells. Considering the significant increase in DAMPs and tumor antigens of cancer cells after QD-Cat-RGD-based RT, we then investigated whether combining RT with QD-Cat-RGD could enhance the recruitment and maturation of DCs. In the results, $\mathrm{DC}\left(\mathrm{CD} 11 \mathrm{c}^{+}\right)$infiltration in tumors was significantly increased in the RT + QD-Cat-RGD group (Supplementary Fig. 11b). DCs infiltrating the tumor site can migrate to the TDLN to present tumor antigens and costimulatory receptors to $\mathrm{T}$ cells, eventually leading to the priming of T cell-mediated antitumor immunity ${ }^{9}$. Therefore, we tested the status of DCs in the TDLN, and the results showed that the costimulatory molecules on DCs $\left(\mathrm{CD} 80^{+} \mathrm{CD} 86^{+} \mathrm{CD} 11 \mathrm{c}^{+}\right)$and antigen presentation molecule on DCs $\left(\mathrm{MHC}-\mathrm{II}^{\text {high }} \mathrm{CD} 11 \mathrm{c}^{+}\right)$ 
a

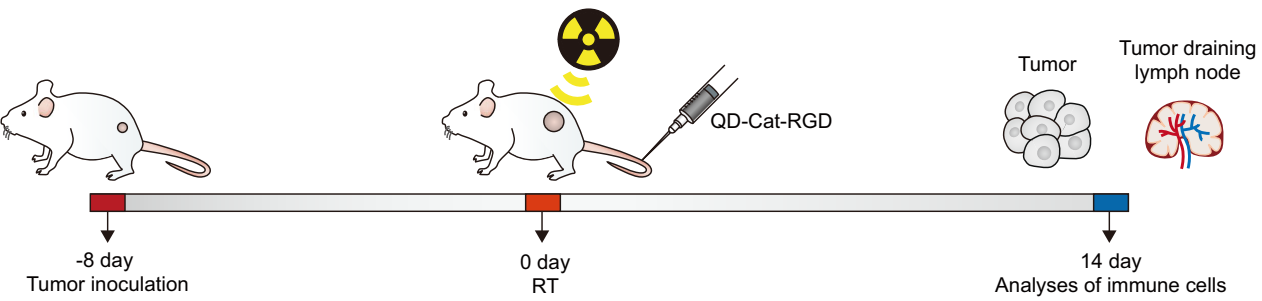

b

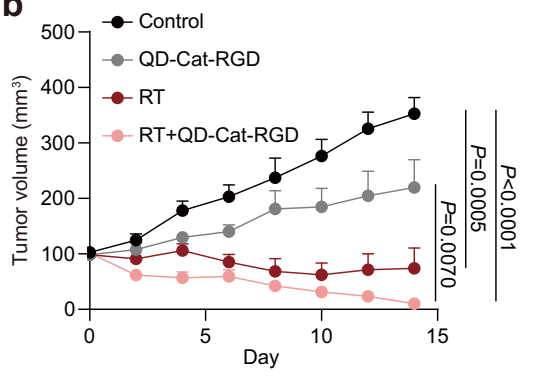

e
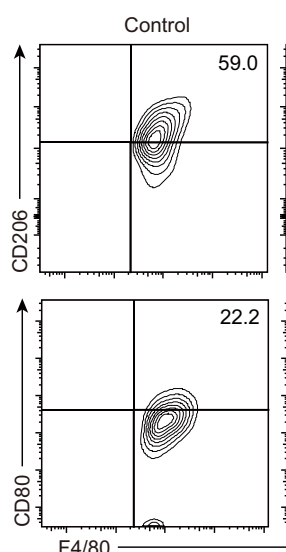

f
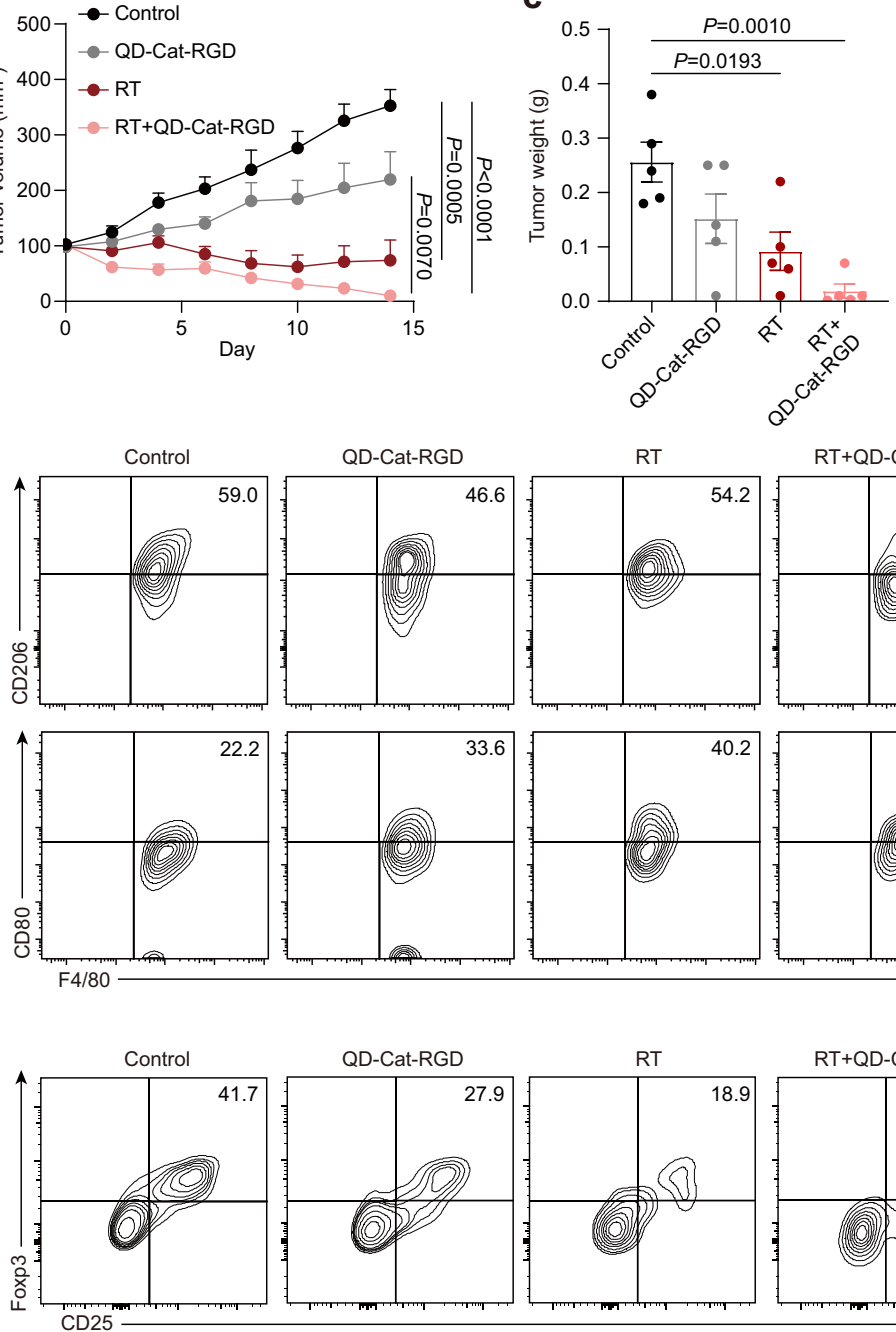

C

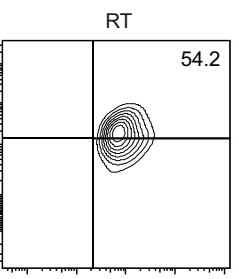

RT+QD-Cat-RGD
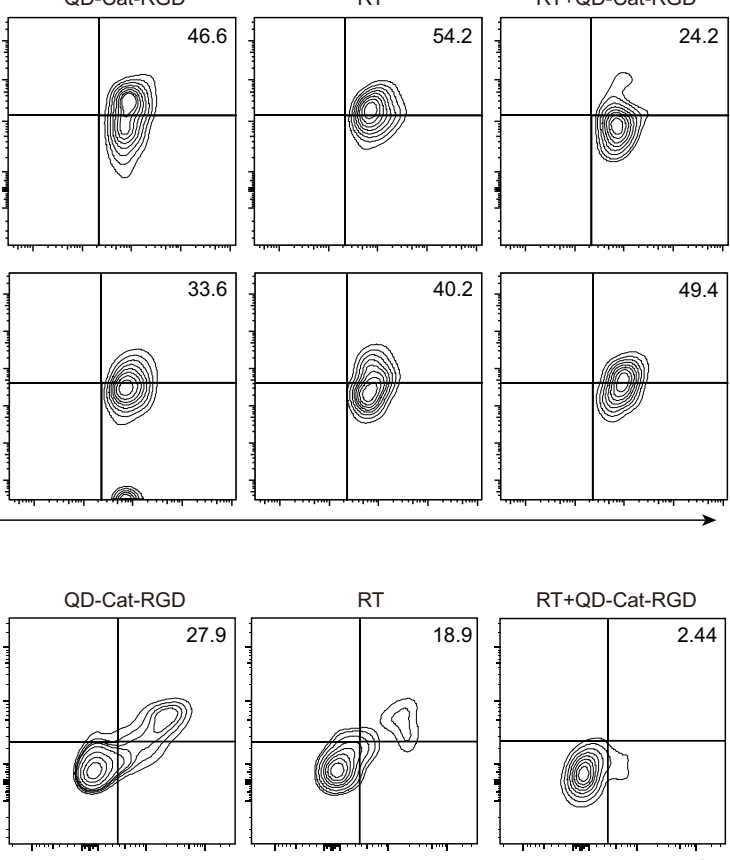

RT+QD-Cat-RGD

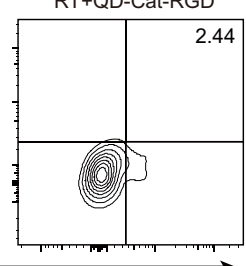

g

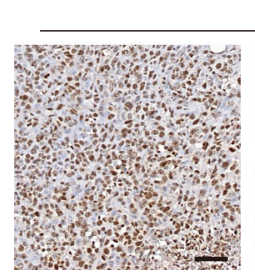

Control

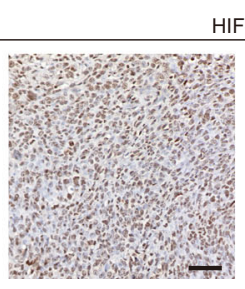

QD-Cat-RGD
HIF-1 $1 \alpha$

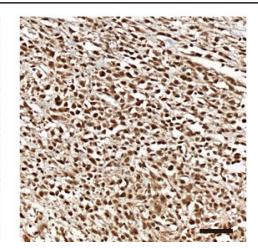

RT

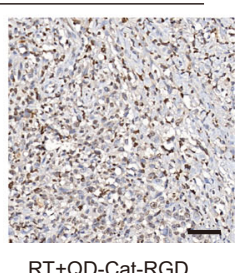

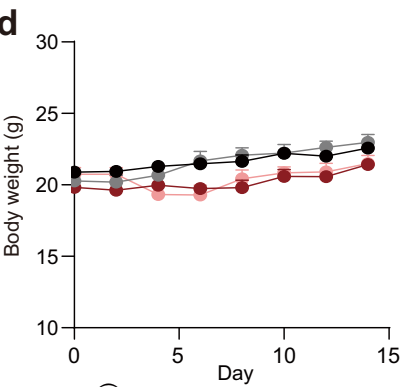
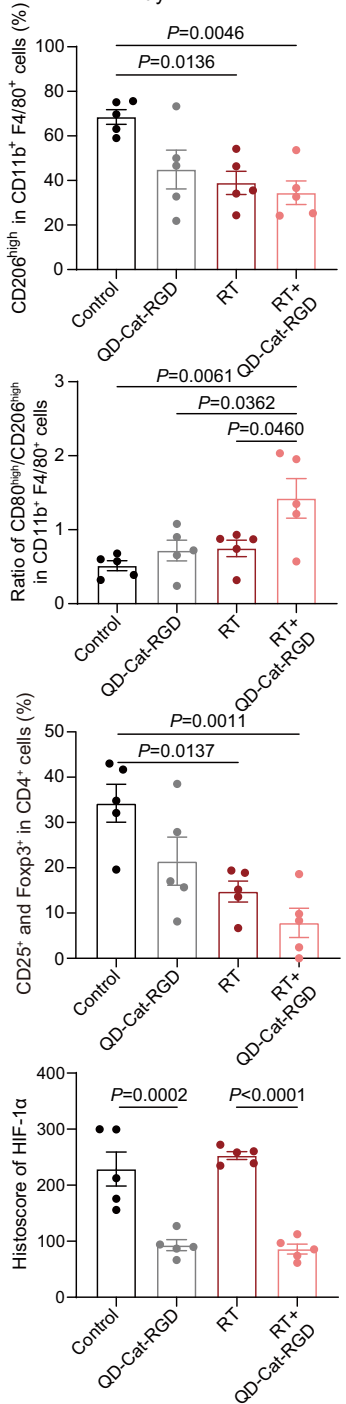

were significantly increased in the TDLN (Fig. 5a, b) after treatment with QD-Cat-RGD-based RT.

$\mathrm{T}$ cells, especially cytotoxic $\mathrm{CD}^{+} \mathrm{T}$ cells, are generally considered to be the main effectors of efficient antitumor immunity triggered by $\mathrm{DCs}^{42}$. With the strong effect on recruitment and activation of DCs, QD-Cat-RGD-based RT consequently caused an increase in tumor-infiltrated $\mathrm{T}$ cells $\left(\mathrm{CD}^{+}\right)$and cytotoxic $\mathrm{CD} 8^{+} \mathrm{T}$ cells $\left(\mathrm{CD}^{+}{ }^{+} \mathrm{CD}^{+}\right)$compared with RT alone (Fig. 5c, d and Supplementary Fig. 11d), which led to a strong $\mathrm{T}$ cell-mediated antitumor immune response ${ }^{43}$. Moreover, the central memory $\mathrm{CD} 8^{+} \mathrm{T}$ cell populations $\left(\mathrm{CD} 8^{+}\right.$ $\left.\mathrm{Tcm}, \mathrm{CD} 44^{+} \mathrm{CD}_{2} 2 \mathrm{~L}^{+} \mathrm{CD} 8{ }^{+}\right)$in the TDLN of the mice in the 
Fig. 4 QD-Cat-RGD-based RT for inhibiting tumor growth, relieving hypoxic conditions and decreasing immunosuppressive cells in the TME. a Schematic showing the experiment using QD-Cat-RGD-based RT to treat mice bearing 4T1 tumors. BALB/c mice ( $n=5$ per group) were implanted subcutaneously with $7 \times 10^{5} 4 \mathrm{~T} 1$ mammary carcinoma cells in the right hind flanks. When the tumor volumes were $100 \mathrm{~mm}^{3}$, mice received different treatments. 4T1 tumors were harvested on day 14. b Tumor growth curves in different groups. Photographs of all tumors and tumor growth curves of tumors from individual mice are shown in Supplementary Fig. 10. c Histogram plot of tumor weight in different groups. $\mathbf{d}$ Body weight curve of different groups. e Representative flow cytometric analysis images of M2-like macrophages (CD206high) and M1-like macrophages (CD80high) gating on F4/ $80+{ }^{+} \mathrm{CD} 11 \mathrm{~b}+\mathrm{CD} 45^{+}$cells. The relative quantification of M2-like macrophages and the ratio of M1/M2 macrophages. f Representative flow cytometric analysis images and relative quantification of Tregs $\left(\mathrm{CD} 25^{+}\right.$and Foxp $\left.3^{+}\right)$gating on $\mathrm{CD} 4{ }^{+} \mathrm{CD} 3^{+} \mathrm{CD} 45^{+}$cells. $\mathbf{g}$ Representative image of immunostaining of $\mathrm{HIF}-1 \alpha$ and relative quantification of the histoscore of immunostaining. Scale bars $=50 \mu \mathrm{m}$. The images of immunostaining ( $\mathbf{g}$ ) were representative of those generated from five mice each group. All data are presented as the mean \pm s.e.m. ( $n=5$ per group) and $n$ represents the number of independent animals. Statistical significance was calculated via one-way ANOVA with Tukey's multiple comparisons test. Source data are provided as a Source Data file.

RT + QD-Cat-RGD group were significantly increased on day 14 after treatment compared with the control group (Fig. 5e), which suggested the potential of conferring durable remission and systemic abscopal effects ${ }^{44,45}$. This evidence demonstrated that treatment combining RT with QD-Cat-RGD could be considered as an immunogenic RT, which promoted maturation and antigen presentation of DCs and consequently led to more effective $\mathrm{T}$ cell-mediated antitumor immunity than RT alone.

To validate the contribution to antitumor effect of cytotoxic $\mathrm{T}$ cells in this combination therapy, $\mathrm{CD} 8^{+} \mathrm{T}$ cells were depleted using antibodies against CD8 (aCD8) in 4T1 tumor-bearing mice treated with QD-Cat-RGD-based RT (Supplementary Fig. 12a). The results demonstrated that the antitumor effects of the combination treatment were abrogated with the depletion of $\mathrm{CD}^{+} \mathrm{T}$ cells (Supplementary Fig. 12b-d). Treatment combining QD-Cat-RGD-based RT with aCD8 caused decreases in tumorinfiltrated T cells $\left(\mathrm{CD}^{+}\right)$and depletion of cytotoxic $\mathrm{CD}^{+} \mathrm{T}$ cells $\left(\mathrm{CD}^{+} \mathrm{CD}^{+}\right)$(Supplementary Fig. 12e,f), confirming that the antitumor effect in primary tumor was mainly induced by $\mathrm{CD}^{+}$ cytotoxic $\mathrm{T}$ cells in the TME.

For exploring the effect from QD-Cat-RGD for alleviating hypoxia and relieving immunosuppression, we added QD-RGD as a control group which was not modified with Cat and had no oxygenation ability. The results showed that QD-Cat-RGD could delay tumor growth (Supplementary Fig. 13a, b) when RT did not apply. However, tumor volumes in the QD-RGD group were not significantly decreased, which implied an antitumor effect of relieving hypoxia by Cat. Furthermore, a significant decrease in the M2-like macrophage infiltration (Supplementary Fig. 13c) and a slight decrease in the Treg infiltration in the TME (Supplementary Fig. 13f) were found in the QD-Cat-RGD group. The intratumoral level of interleukin-10 (IL-10), an immunosuppressive cytokine predominantly secreted by M2 macrophages, was also found to decrease in the QD-Cat-RGD group (Supplementary Fig. 13g). DC and T cell infiltrations were found to increase in the TME after treatment with QD-Cat-RGD (Supplementary Fig. 13j, k). These results revealed that Cat modified on QDs could independently play a role in inhibiting tumor growth by reducing immunosuppressive cell infiltration and increasing $\mathrm{T}$ cell infiltration in the TME. These findings were consistent with previous reports demonstrating that alleviating hypoxia could reduce the infiltration of immunosuppressive cells in the TME, which delayed tumor growth ${ }^{26,46,47}$. We next evaluated the synergistic antitumor effect of alleviating immunosuppression by relieving hypoxia and reinforcing RT efficiency by QD-Cat-RGD nanoprobes when combined with RT. We added a group that combined RT with QD-Cat (without Cat modification) and a group that QD-Cat-RGD injection was after RT treatment as comparable groups to explore the synergistic effect in suppressing tumor growth. The combination of alleviating immunosuppression and reinforcing RT efficiency exhibited a synergistic effect in suppressing tumor growth (Supplementary
Fig. 13d). RT + QD-Cat-RGD (after RT injection) and RT + QDRGD slightly suppressed tumor growth but no significance was found when compared with the RT group (Supplementary Fig. 13d). M2-like macrophage infiltration and intratumoral level of IL-10 in the TME decreased after treatment with QD-CatRGD-based RT (Supplementary Fig. 13e, i). Moreover, DCs, $\mathrm{T}$ cells, and $\mathrm{CD}^{+}{ }^{+}$cytotoxic $\mathrm{T}$ cells in the TME were significantly increased in the RT + QD-Cat-RGD group (Supplementary Fig. 13l, m, o). These results suggested that Cat and high-Z element $\mathrm{Pb}$ in QD-Cat-RGD exhibited a synergistic effect in RT for suppressing tumor growth through inhibiting immunosuppression and facilitating cytotoxic $\mathrm{T}$ cell-mediated antitumor immunity.

The abscopal effect boosted by nanoprobe-mediated RT and ICB. ICD driven by RT and the activation of a tumor-specific T cell-dependent immune response result in the regression or stabilization of distant untreated tumors (the abscopal effect). Many studies indicate that RT provides an opportunity to increase abscopal response rates when combined with ICB against checkpoints such as programmed cell death 1 (PD-1) and cytotoxic T-lymphocyte-associated protein 4 (CTLA-4) ${ }^{48,49}$. However, the abscopal effect can still be stifled by hypoxic conditions and immunosuppression in the TME even when combining RT with immunotherapy ${ }^{11}$. Several types of immunosuppressive cells infiltrating the TME before or after RT can limit the abscopal response ${ }^{11}$. Considering that QD-Cat-RGD had the capabilities of relieving hypoxia and decreasing tumor infiltrating immunosuppressive cells as well as increasing $\mathrm{CD} 8^{+} \mathrm{Tcm}$ in the TDLN, we further investigated the efficacy of the QD-Cat-RGD nanoprobe for inducing robust abscopal effects.

To investigate whether QD-Cat-RGD survived and reinforced the abscopal effect induced by localized RT combined with immunotherapy, we used an antibody against PD-1 (aPD-1) as an ICB to reveal this process. Then, we built a metastatic tumor model to reveal the abscopal effect. $4 \mathrm{~T} 1$ cancer cells were first injected into the right hind flank of mice on day -8 as primary tumors and subsequently injected into the left hind flanks on day -6 as a distant tumor mimic (Fig. 6a). On day 0 , the mice were intravenously injected with QD-Cat-RGD $\left(150 \mu \mathrm{L}, 2 \mathrm{mg} \mathrm{mL}^{-1}\right)$, and the primary tumors were locally treated with RT (12 Gy) at $2 \mathrm{~h}$ p.i. Then, the mice were treated with aPD- 1 on day 1,3 , and 5 (Fig. 6a). Tumor growth curves showed that the primary tumor (right side) was inhibited by QD-Cat-RGD-based RT combined with aPD-1 treatment (Fig. 6b and Supplementary Fig. 14). Additionally, the growth of distant tumors (left side) was inhibited (Fig. 6c and Supplementary Fig. 15). Consistent with these results, the numbers of intratumoral $\mathrm{T}$ cells $\left(\mathrm{CD}^{+}\right)$and cytotoxic $\mathrm{T}$ cells $\left(\mathrm{CD} 8^{+} \mathrm{CD} 3^{+}\right)$were significantly increased in distant tumors of the RT + QD-Cat-RGD + aPD-1 group (Fig. 6d-f). Granzyme B, interferon- $\gamma($ IFN- $\gamma$ ), and tumor necrosis factor- $\alpha$ (TNF- $\alpha)$, cytotoxic effectors mainly expressed 
a

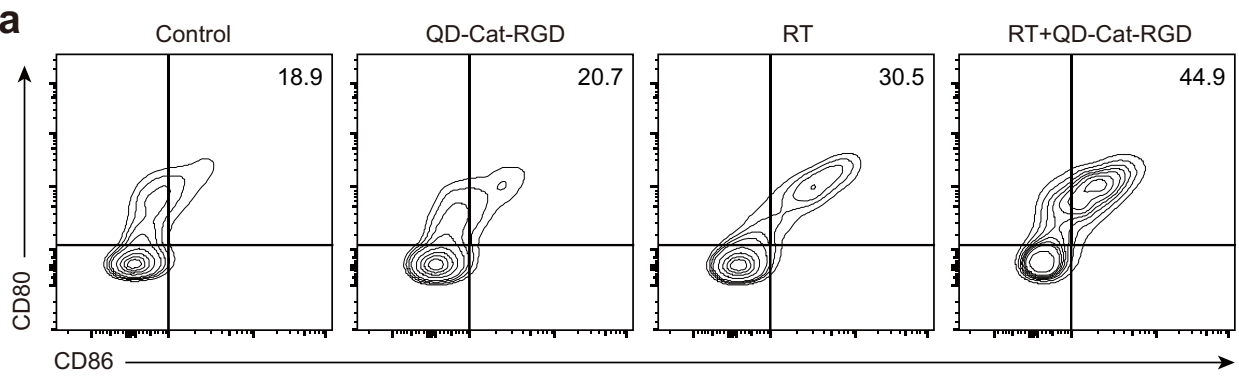

b

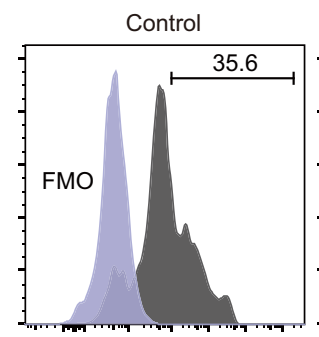

MHC-II

C

$\uparrow_{\substack{c \\ 0 \\ \infty}}^{c}$

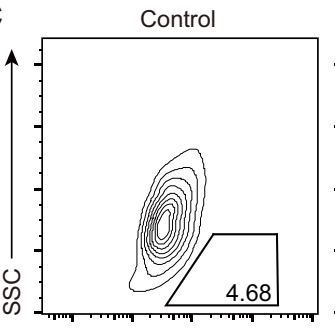

$\mathrm{CD} 3$

d

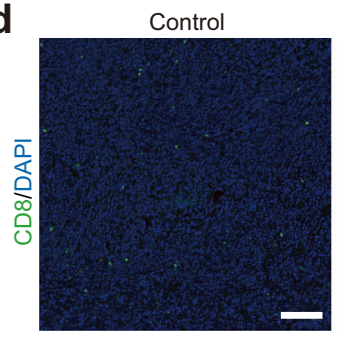

e


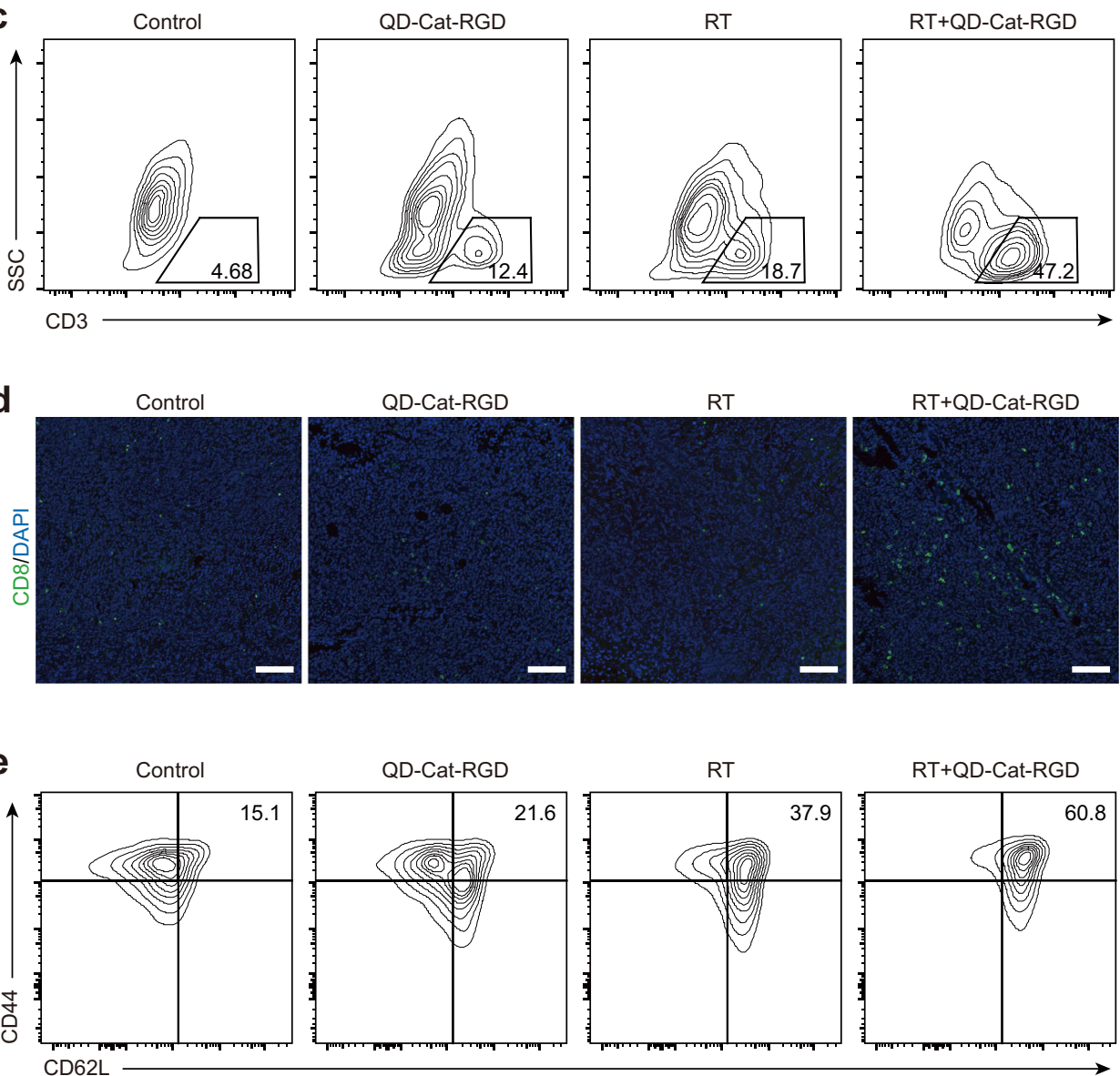

QD-Cat-RGD

RT

RT+QD-Cat-RGD
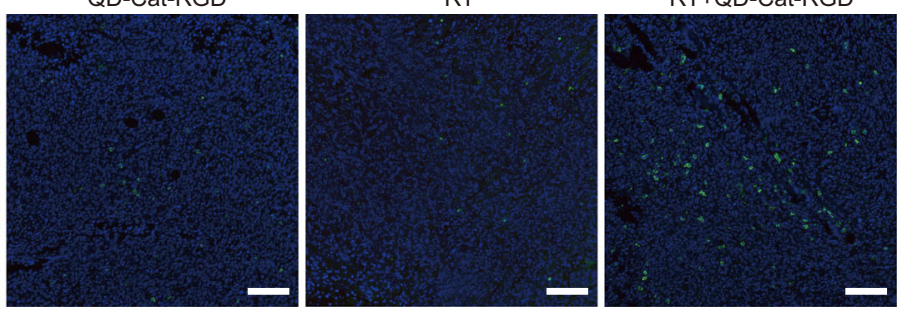

QD-Cat-RGD

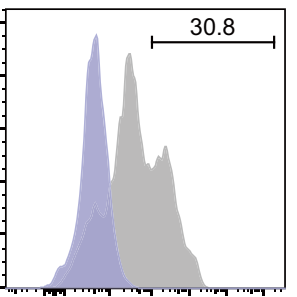

1
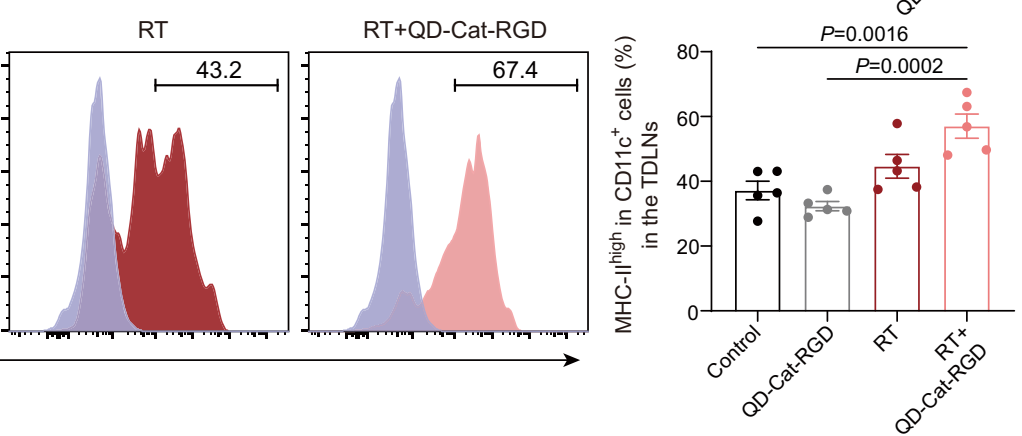
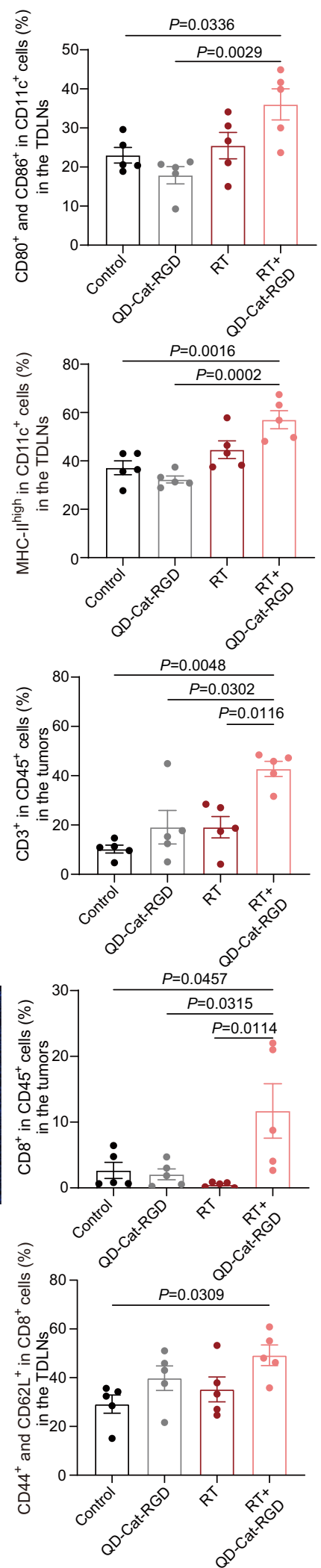

by cytotoxic $\mathrm{T}$ cells, were also increased in distant tumors of the $\mathrm{RT}+\mathrm{QD}-\mathrm{Cat}-\mathrm{RGD}+\mathrm{aPD}-1$ group, as measured by IHC and ELISA (Fig. 6g-i). We further investigated the mechanism contributing to the suppression of distant tumor. Encouraged by the results that QD-Cat-RGD could relieve hypoxic condition and reduce the infiltration of immunosuppressive cells in TME

which could hinder antitumor immunity mediated by cytotoxic $\mathrm{T}$ cells ${ }^{50,51}$, we tested the changes of Tregs, M2-like macrophages, and IL-10 in the TME of distant tumor. QD-Cat-RGD could also work in distant tumors and the intratumoral M2-like macrophages, Tregs, and the level of IL-10 were decreased in distant tumors in the $\mathrm{RT}+\mathrm{QD}-\mathrm{Cat}-\mathrm{RGD}+\mathrm{aPD}-1$ group 
Fig. 5 QD-Cat-RGD-based RT for promoting DC maturation and antigen presentation in TDLNs and consequently inducing $T$ cell-driven antitumor immunity. a Representative flow cytometric analysis images and relative quantification of co-stimulatory molecules $\mathrm{CD}^{2} 0^{+} \mathrm{CD} 86^{+}$gating on $\mathrm{CD} 45^{+}$ $\mathrm{CD}_{11} \mathrm{c}^{+}$cells in TDLNs. b Representative flow cytometric analysis images and relative quantification of MHC-II expression on CD11c ${ }^{+}$CD45 ${ }^{+}$cells in the TDLNs. c Representative flow cytometric analysis images and relative quantification of $C D 3^{+} \mathrm{T}$ cells gating on $\mathrm{CD} 45^{+}$in tumors. $\mathbf{d}$ Representative immunofluorescence images of tumors showing $\mathrm{CD} 8^{+} \mathrm{T}$ cells and relative quantification of $\mathrm{CD} 8^{+} \mathrm{CD} 3^{+} \mathrm{T}$ cells gating on $\mathrm{CD} 45^{+}$cells in tumors by flow cytometry. Scale bars $=50 \mu \mathrm{m}$. The immunofluorescent images were representative of those generated from three mice each group. e Representative flow cytometric analysis images and relative quantification of $\mathrm{CD} 44^{+} \mathrm{CD} 62 \mathrm{~L}^{+} \mathrm{Tcm}$ cells gating on $\mathrm{CD} 8{ }^{+} \mathrm{CD} 3^{+} \mathrm{CD} 45^{+}$cells in the TDLNs. All data are presented as the mean \pm s.e.m. ( $n=5$ per group) and $n$ represents the number of independent animals. Statistical significance was calculated via one-way ANOVA with Tukey's multiple comparisons test. FMO fluorescence-minus-one. TDLN tumor-draining lymph node. Source data are provided as a Source Data file.

(Supplementary Fig. 16a-c). This evidence demonstrated that QD-Cat-RGD-based RT combined with aPD-1 could intensely boost the abscopal effect and inhibit the growth of distant tumors.

Considering that tumor metastasis is responsible for approximately $90 \%$ of cancer-related mortality, preventing the spread of metastasis is beneficial for prolonging the overall survival of patients $^{52}$. Encouraged by the increase in the abscopal effect in the subcutaneous tumor model, which implied strong systemic antitumor immunity, we evaluated this therapeutic strategy to inhibit early metastasis and prolong survival using an experimental model of metastasis at an early stage ${ }^{53-55}$. To establish the model of metastasis at an early stage, 4T1 cancer cells stably transfected with the luciferase gene (4T1-Luc) were injected into the right hind flank of mice as primary tumors. When the volume of the primary tumors reached approximately $100 \mathrm{~mm}^{3}$, 4T1-Luc cancer cells were then intravenously injected into the mice to mimic the escape of tumor cells from the primary tumor site into the circulation at an early stage and seeding in distant organs especially the lung. On the following day, the mice were intravenously injected with PBS or QD-Cat-RGD and then underwent surgery or RT to eliminate the primary tumors. Next, the mice were treated with aPD-1 on days 1,3 , and 5 (Fig. 7a). The growth of metastatic tumors was monitored by bioluminescence imaging. The results showed that lung metastases were found in most mice treated with surgery, even those mice treated with QD-Cat-RGD, aPD-1, or the combination treatment of QDCat-RGD and aPD-1 (Fig. 7b). In contrast, mice treated with QDCat-RGD-based RT exhibited a significantly slowed tumor growth of the lung metastases, while the combination with aPD-1 further reduced lung metastases (Fig. 7b). The survival analysis showed that $80 \%$ of mice could survive for 90 days in the RT + QD-Cat-RGD + aPD-1 group (Fig. 7c). Only 20\% of mice survived for 90 days in the surgery + QD-Cat-RGD + aPD-1, $\mathrm{RT}+\mathrm{QD}-$ Cat-RGD, and $\mathrm{RT}+\mathrm{aPD}-1$ groups (Fig. 7c). The survival rate of mice in the control, QD-Cat-RGD, aPD-1, QDCat-RGD + aPD-1, and RT groups reached $0 \%$ before day 50 . These results further confirmed that QD-Cat-RGD-based RT combined with immunotherapy could efficiently inhibit metastasis at an early stage and prolong overall survival.

Combination therapy against B16F10 melanoma. Based on the above results, QD-Cat-RGD-based RT could induce an effective antitumor immune response in the $4 \mathrm{~T} 1$ breast cancer model. Therefore, we wondered whether QD-Cat-RGD could potentially be used for other tumor types. Based on the targeting ability of RGD, we chose the B16F10 (integrin $\alpha_{v} \beta_{3}$-positive) murine melanoma model as the next tested model ${ }^{35}$. In the B16F10 tumor-bearing mouse model, we found that tumor growth was inhibited by the combination of RT and QD-Cat-RGD (Fig. 7d and Supplementary Fig. 17), consistent with the results obtained from the $4 \mathrm{~T} 1$ breast cancer model. In addition, the QD-CatRGD-based RT resulted in significantly prolonged survival of mice relative to mice treated with PBS (control), QD-Cat-RGD, and RT (Fig. 7e). These results indicated that the combination strategy of QD-Cat-RGD-based RT can be extended to other types of tumors.

The cytotoxicity and biosafety of QD-Cat-RGD. We next evaluated the potential cytotoxicity and biosafety of QD-CatRGD nanoprobes. The cytotoxicity of QD-Cat-RGD was measured by Cell Counting Kit-8 (CCK8) assay. The viability of 4T1 cancer cells and normal human immortalized oral epithelial cells (HIOECs) was not significantly affected after incubation with different concentrations of QD-Cat-RGD for $8 \mathrm{~h}$ (Supplementary Fig. 18). We also investigated the in vivo biosafety of the injected nanoprobes. $H \& E$ staining results for the hearts, livers, spleens, lungs, and kidneys in treated mice showed no abnormality in cellular morphology compared with control mice (Supplementary Fig. 19). We additionally performed routine blood tests and blood biochemical tests 10 days after intravenous injection of the QDCat-RGD nanoprobes. Routine blood tests and functional indicators of the liver and kidney, including alanine aminotransferase (ALT), aspartate aminotransferase (AST), carbamide (UREA), and creatinine (CREA), showed no significant difference between the control group and the QD-Cat-RGD injection group (Supplementary Table 1). These results demonstrated that the welldesigned QD-Cat-RGD showed no observable cytotoxicity or in vivo toxicity.

\section{Discussion}

$\mathrm{RT}$ is closely associated with the role of the immune system in the treatment of tumors ${ }^{8}$. At present, an increasing number of studies on RT and nanotechnology have gradually focused on influencing individual's immune system to treat cancer ${ }^{56-59}$. In addition, several studies claimed that decomposing hydrogen peroxide to relieve hypoxia results in increased the radiosensitivity of cancer cells and weakens the immunosuppressive effect in the TME ${ }^{24-26}$ Recently, the combination of RT and immune checkpoint therapies resulted in almost complete regression of primary tumors and inhibited the development of distant tumors. However, there are still a number of patients who are not responsive or sensitive to this treatment strategy. An important mechanism of radioresistance is due to various inhibitory immune cells in the TME, such as Tregs and M2-like macrophages. Therefore, we urgently need a platform to simultaneously enhance the efficiency of RT and inhibit the immunosuppressive TME to boost antitumor immunity and the systemic abscopal effect caused by the combination of RT and immunotherapy.

In this work, a theranostic nanoprobe was developed by using NIR-IIb emitting QDs for the radioimmunotherapy of cancer. The NIR-IIb emitting and targeting ability of QD-Cat-RGD nanoprobes enabled the in vivo imaging with a high resolution, which could clearly discriminate the margin of the tumor from the surrounding tissues to guide the range of the tumor in RT. Moreover, the QDCat-RGD nanoprobe was able to enhance the radiotherapeutic efficacy and immunogenicity of cancer cells via ICD, resulting in a robust immune response after RT. Cat coated on the nanoprobe 
a

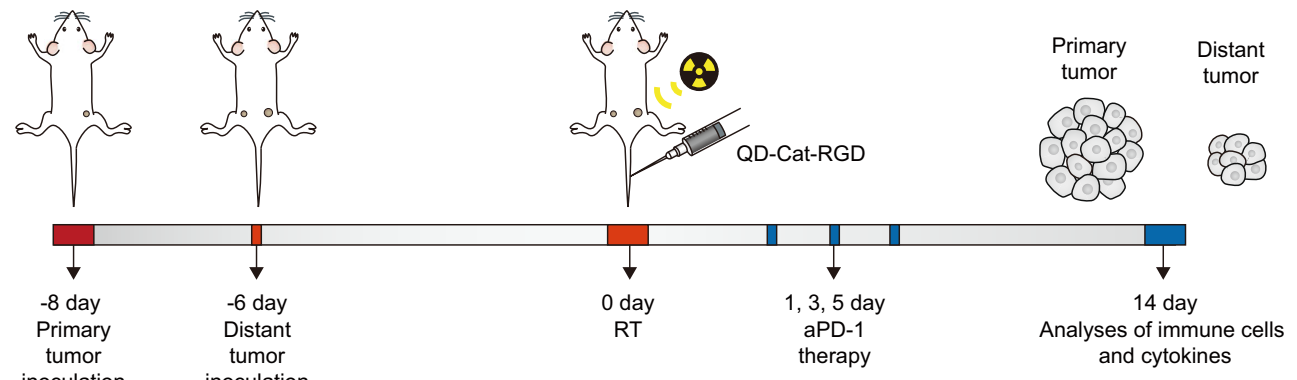

b

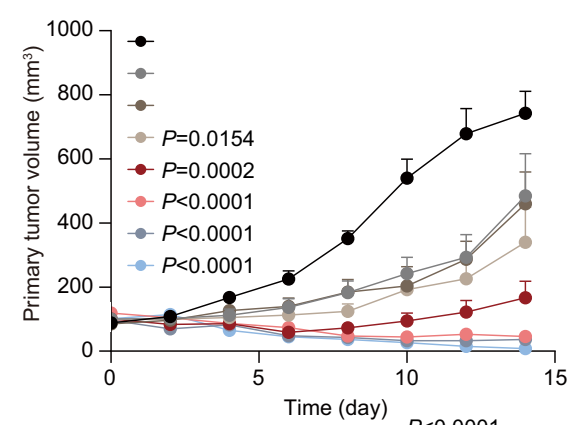

d

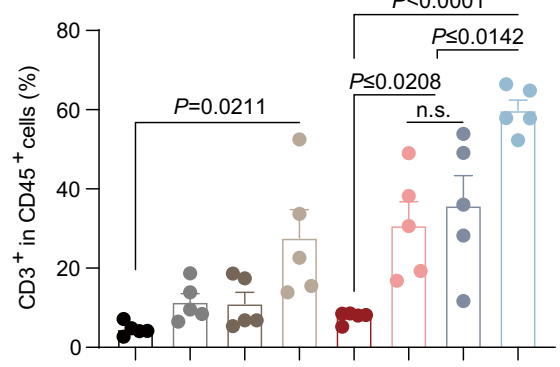

c
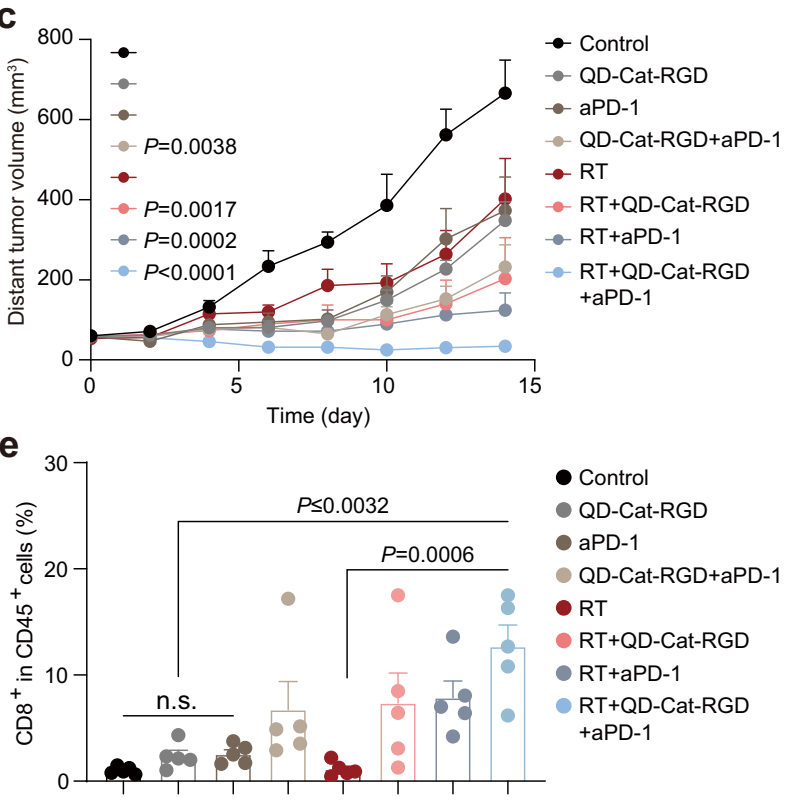

$R T+Q D-C a t-R G D$

f

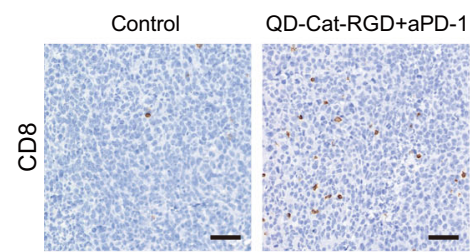

g

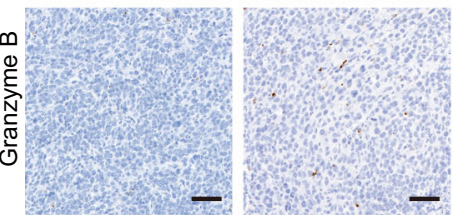

RT
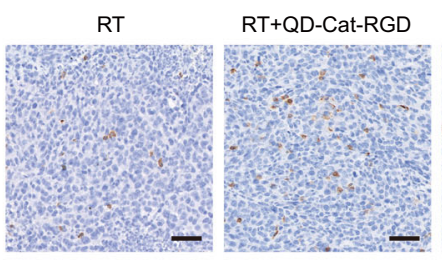

RT+aPD-1 $+\mathrm{aPD}-1$
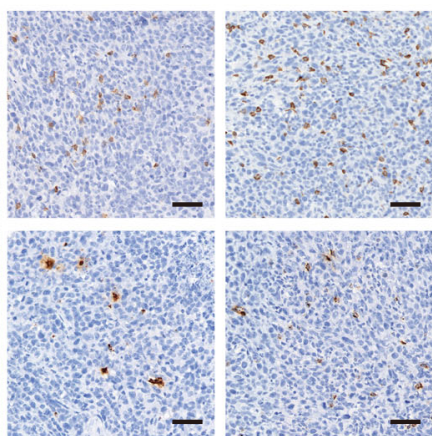

h
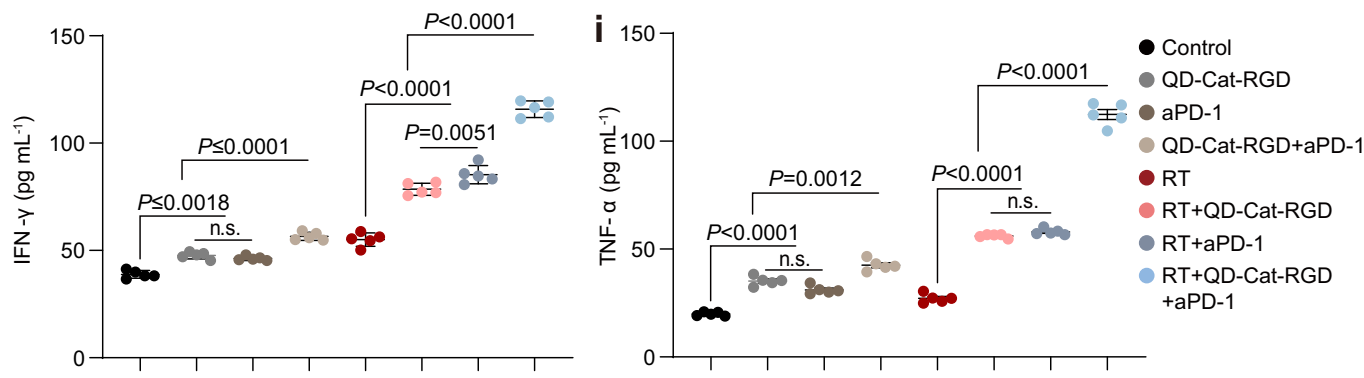

could relieve hypoxic conditions in the tumor site to enhance the radiosensitization of cancer cells and reduce the immunosuppressive TME, which is considered to be a major obstacle for antitumor immunity. In addition, combining nanoprobe-mediated RT with immunotherapy could remarkably boost the abscopal effect leading to a systemic antitumor immune response and elimination of distant metastases. The potential toxic elements in QD-Cat-RGD may limits the clinical transformation of QD-Cat-RGD in RT. This strategy still provides good prospects for the future RT to enhance the therapeutic precision and efficacy of RT and promote antitumor immune response, prolonging survival and improving the quality of life of patients with cancer. 
Fig. 6 QD-Cat-RGD-based RT in combination with aPD-1 for boosting the abscopal effect. a Schematic showing the experiment using QD-Cat-RGDbased RT combined with aPD-1 to treat mice bearing 4 T1 tumors on both sides. The tumor on the right side was designated as the primary tumor for in situ radiation therapy, and the tumor on the left side was designated as a distant tumor. BALB/c mice ( $n=5$ per group) were implanted subcutaneously with $7 \times 10^{5} 4 \mathrm{~T} 1$ mammary carcinoma cells in the right hind flanks and after 2 days consequently implanted subcutaneously with $7 \times 10^{5} 4 \mathrm{~T} 1$ cells on left hind flanks. When the primary tumor volumes were $100 \mathrm{~mm}^{3}$, the mice received different treatments. 4T1 tumors were harvested on day 14 . b Tumor growth curves of primary tumors on the right side in the different groups. The $P$ values are shown to compare with the control group. Photographs of all primary tumors and tumor growth curves of tumors from individual mice are shown in Supplementary Fig. 14. c Tumor growth curves of distant tumors on the left side in the different groups. The $P$ values are shown to compare with the control group. Photographs of all distant tumors and tumor growth curves of tumors from individual mice are shown in Supplementary Fig. 15. d Quantification of flow cytometric analysis of CD3 ${ }^{+} \mathrm{T}$ cells gating on CD45+ in the distant tumors of the different groups. e Quantification of flow cytometric analysis of CD8 ${ }^{+} \mathrm{CD} 3^{+} \mathrm{T}$ cells gating on $\mathrm{CD} 45^{+}$in distant tumors of the different groups. $\mathbf{f}, \mathbf{g}$ Representative image of immunostaining of CD8 and granzyme B in distant tumors in the different groups. Scale bars $=50 \mu \mathrm{m}$. The images of immunostaining $(\mathbf{f}, \mathbf{g})$ were representative of those generated from three mice each group. $\mathbf{h}, \mathbf{i}$ Cytokine levels of IFN- $\gamma$ and TNF- $\alpha$ in distant tumors after different treatments as measured by ELISA. All data except $\mathbf{f}$ and $\mathbf{g}$ are presented as the mean \pm s.e.m. ( $n=5$ per group) and $n$ represents the number of independent animals. Statistical significance was calculated via one-way ANOVA with Tukey's multiple comparisons test. n.s. not significant. Source data are provided as a Source Data file.

\section{Methods}

Chemicals. Lead chloride $\left(\mathrm{PbCl}_{2}, 99 \%\right.$, powder $)$ and cadmium oxide $(\mathrm{CdO}$, $99.99 \%$, power) were purchased from Alfa. Sulfur (S, $99.999 \%$, powder) was purchased from Adamas. Oleylamine (OLA, technical grade, 70\%), oleic acid (OA, technical grade, 90\%), 1-octadecene (ODE), 2-(N-morpholino) ethanesulfonic acid (MES) hydrate, poly(acrylic acid) (Mw 1,800), N,N'-dicyclohexylcarbodiimide (DCC), $\mathrm{N}$-(3-dimethylaminopropyl)- $\mathrm{N}^{\prime}$-ethylcarbodiimide hydrochloride (EDC), and catalase (Cat) were purchased from Sigma-Aldrich. Phosphate buffered saline (PBS) was purchased from Hyclone. 8-Arm PEG-amine (Mw $40 \mathrm{~K}$ ) was purchased from Advanced BioChemicals. The cyclo(RGDyK) (97\%) was purchased from China Peptides. Other reagents were of analytical grade. Ultrapure water was used in all needed experiments.

Synthesis of PbS/CdS QDs. PbS/CdS core-shell quantum dots (QDs) were prepared as previously described ${ }^{30}$. S $(5 \mathrm{mmol}, 0.08 \mathrm{~g})$ and OLA $(7.5 \mathrm{~mL})$ were mixed in a two-neck flask at $120^{\circ} \mathrm{C}$ for $30 \mathrm{~min}$ under a vacuum of $0.1 \mathrm{MPa} . \mathrm{PbCl}_{2}$ $(3 \mathrm{mmol}, 0.834 \mathrm{~g})$ and OLA $(7.5 \mathrm{~mL})$ were mixed in a three-neck flask at $120^{\circ} \mathrm{C}$ for $30 \mathrm{~min}$ and then heated to $160^{\circ} \mathrm{C}$ under a vacuum of $0.1 \mathrm{MPa}$. The $\mathrm{S}$ and $\mathrm{PbCl}_{2}$ were dissolved completely to form S-OLA and Pb-OLA. S-OLA (2.25 mL) was quickly injected into the $\mathrm{Pb}$-OLA under stirring at $160^{\circ} \mathrm{C}$ and kept the condition for $1 \mathrm{~h}$. Subsequently, the temperature was quickly cooled down to room temperature, and the growth reaction was terminated by introducing hexane $(10 \mathrm{~mL})$ into the reaction solution. The PbS QDs were separated and purified by adding excess ethanol and OA, collected via centrifugation. After centrifugation of the suspension and decantation of the supernatant, the PbS QDs were re-suspended in ODE. Next, CdO $(9.2 \mathrm{mmol}, 1.2 \mathrm{~g})$, OA $(8 \mathrm{~mL})$, and ODE $(20 \mathrm{~mL})$ were heated to $200^{\circ} \mathrm{C}$ under a vacuum of $0.1 \mathrm{MPa}$. Subsequently, the temperature cooled down to $100^{\circ} \mathrm{C}$ and stabled for $30 \mathrm{~min}$, PbS QDs $(5 \mathrm{~mL})$ was bubbled with Ar for $5 \mathrm{~min}$, and then injected into the Cd precursor under stirring at $100^{\circ} \mathrm{C}$ and kept the condition for $30 \mathrm{~min}$. The growth reaction was terminated by introducing hexane $(5 \mathrm{~mL})$ into the reaction solution. $\mathrm{PbS} / \mathrm{CdS} \mathrm{QDs}$ were precipitated with excess ethanol and then re-dispersed in hexane.

Preparation of QD-Cat-RGD nanoprobes. The as-synthesized QDs were modified as previously described ${ }^{60}$. Polyacrylic acid (average $\mathrm{Mw} \sim 1,800,0.9 \mathrm{~g}$ ) and DCC $(1.56 \mathrm{~g})$ were mixed into a round-bottom flask. N,N-dimethylformamide (DMF) $(10 \mathrm{~mL})$ was added to dissolve the mixture. Subsequently, OLA $(1.2 \mathrm{~mL}$, molar ratio of OLA to PAA is $30 \%$ ) were added dropwise into the reaction flask. The mixture solution was stirred overnight, and $0.5 \mathrm{M} \mathrm{HCl}(50 \mathrm{~mL})$ were added to the mixture solution. The precipitate was isolated by centrifugation and re-dissolved in methanol $(3 \mathrm{~mL})$. Then, $1 \mathrm{M} \mathrm{HCl}(20 \mathrm{~mL})$ was added to the solution and the precipitate was isolated by centrifugation. The precipitate was dissolved in chloroform $(5 \mathrm{~mL})$ and washed by $1 \mathrm{M} \mathrm{HCl}(10 \mathrm{~mL})$. Collecting and drying over the organic phase with anhydrous $\mathrm{Na}_{2} \mathrm{SO}_{4}$. Under a vacuum, chloroform was removed and the oleyaminebranched poly(acrylic acid) (OPA) was collected (with an average Mw of $\sim 3,000$ determined by gel permeation chromatography). Subsequently, PbS/CdS QDs $(5.0 \mathrm{mg})$ were added to mixture solution $(15 \mathrm{mg}$ OPA dissolved in $2.0 \mathrm{~mL}$ chloroform). The mixture was stirred for $30 \mathrm{~min}$ at room temperature and the solvent was removed by a rotary evaporator under a vacuum. Under the sonication, the products were dissolved completely in $2 \mathrm{~mL}$ of $50 \mathrm{mM}$ sodium carbonate solution. The QDs were precipitated for $1 \mathrm{~h}$ at $416,000 \mathrm{~g}$ by ultracentrifuge, and dissolved in $2 \mathrm{~mL} \mathrm{pH} 8.5$ MES buffer $(0.01 \mathrm{M})$. The 8-Arm PEG-amine molecules, RGD peptides, and Cat were dissolved in $500 \mu \mathrm{L}$ pH $8.5 \mathrm{MES}$ buffer $(0.01 \mathrm{M})$ and gradually added to the OPAmodified QD solution with stirring. EDC $(15 \mathrm{mg})$ were dissolved in $150 \mu \mathrm{L} \mathrm{pH} 8.5$ MES buffer $(0.01 \mathrm{M})$, and gradually added to the mixture solution with stirring and reacted overnight. The QD-Cat-RGD nanoprobes were precipitated with ultracentrifugation at $416,000 \mathrm{~g}$ for $30 \mathrm{~min}$ to remove excess reactants. The precipitate was dissolved in $1 \times \mathrm{PBS}$ and stored at $4{ }^{\circ} \mathrm{C}$.
Nanoprobe characterization. TEM images were collected using a JEM-2100F electron microscope (JEOL) (operating at a $200 \mathrm{kV}$ accelerating voltage). The zeta potentials and DLS data were recorded on a Malvern Nano-ZS ZEN3600 zetasizer. EDS data were obtained by using an EDX spectrometry (EDAX Inc.) equipped on a JEM 2010F microscope. The generation of $\mathrm{O}_{2}$ was measured by an oxygen probe (JPBJ-608 portable Dissolved Oxygen Meters, Shanghai INESA Scientific Instrument Factory). The spectra were obtained from a Lambda $750 \mathrm{~S}$ UV-Vis-NIR spectrometer (Malvern). Photoluminescence spectra were measured using a FLS1000 fluorescence spectrometer with a Xenon lamp exciter and a PM1700 near infrared photomultiplier detector (Edinburgh Instruments).

Fluorescence imaging of cells and animals in the NIR-Ilb window. For NIR-IIb imaging, cells were coincubated with QD-Cat $\left(300 \mu \mathrm{g} \mathrm{mL}^{-1}\right)$ or QD-Cat-RGD $\left(300 \mu \mathrm{g} \mathrm{mL}^{-1}\right)$ in PBS for $30 \mathrm{~min}$ followed by washing with PBS three times. The cells were fixed with $4 \%$ paraformaldehyde for $30 \mathrm{~min}$. Finally, cells in the NIR-IIb window were imaged by a $\times 10$ Mitutoyo Plan Apo NIR infinity corrected objective (Edmund Optics). Fluorescence images in the NIR-IIb window were imaged by a two-dimensional InGaAs camera (NIR vana, Princeton Instruments) and LightField software v.6.4 (Princeton Instruments) ${ }^{31,61}$

For mouse NIR-IIb imaging, 4T1 tumor-bearing mice were intravenously injected with $150 \mu \mathrm{L}$ QD-Cat-RGD $\left(2 \mathrm{mg} \mathrm{mL}^{-1}\right)$. NIR-IIb fluorescence images of the mouse body were recorded by a NIR-II small animal InGaAs camera (NIR vana, Princeton Instruments). The excitation power density was 25 or $50 \mathrm{~mW} \mathrm{~cm}^{-2}$ provided by an $808 \mathrm{~nm}$ laser as previously described ${ }^{31,61}$. The exposure time for NIR-IIb fluorescence imaging was $200 \mathrm{~ms}$.

In vivo pharmacokinetics and biodistribution of QDs. The $4 \mathrm{~T} 1$ tumor-bearing mice intravenously injected with QD-Cat-RGD $\left(150 \mu \mathrm{L}, 2 \mathrm{mg} \mathrm{mL}^{-1}\right)$ or QD-Cat (without RGD) $\left(150 \mu \mathrm{L}, 2 \mathrm{mg} \mathrm{mL}^{-1}\right)$ nanoprobes were used for pharmacokinetics and biodistribution studies. At $0.5,1,3,8,24,48$, and $72 \mathrm{~h}$ p.i., $50 \mu \mathrm{L}$ blood from treated mouse was collected. By using ICP-OES to calculate the concentration of $\mathrm{Pb}$ in blood to estimate the percentage of the QD-Cat-RGD in blood. The tissues including spleen, liver, lung, kidney, and tumor were collected, weighed, and then dissolved completely in $5 \mathrm{~mL}$ digest solution $\left(\mathrm{HNO}_{3}: \mathrm{H}_{2} \mathrm{O}_{2}=4: 1\right)$ for $2 \mathrm{~h}$ until the solution turned to clear Subsequently, the solutions were diluted by ultrapure water to $10 \mathrm{~mL}$ and analyzed by ICP-OES to measure the concentration of $\mathrm{Pb}$ in the resulting solutions. The calculation formula of $\% \mathrm{ID} \mathrm{g} \mathrm{g}^{-1}$ is (the amount of $\mathrm{Pb}$ element in organs) $\times$ (the amount of $\mathrm{Pb}$ element in ID $\times$ the weight of organs $)^{-1} \times 100 \%$. The pharmacokinetic parameters were calculated by the software PKSolver (v. 2.0 $)^{62}$.

Cellular experiments. The murine breast cancer cell line 4T1 (Cat CRL-2539) and the murine melanoma cell line B16F10 (Cat CRL-6475) were purchased from the American Type Culture Collection (ATCC). HIOEC was a gift from the Shanghai Ninth People's Hospital. 4T1 and B16F10 cells were cultured in RPMI 1640 medium modified (HyClone) containing 10\% FBS (Gibco) and $1 \%$ penicillin (HyClone). HIOEC cells were cultured in serum-free keratinocyte medium (Gibco). For hypoxia exposure, cells were incubated under hypoxic conditions $\left(37^{\circ} \mathrm{C}, 1 \% \mathrm{O}_{2}, 5 \% \mathrm{CO}_{2}\right)$ using an incubator (Galaxy $170 \mathrm{R}$, Eppendorf). Clonogenic assays of cells in vitro were performed as previously described ${ }^{63}$. For clonogenic assays, cells were seeded in six-well plates $(2,000$ cells per well). Then, cells under hypoxic conditions were inoculated with QD-Cat-RGD $\left(50 \mu \mathrm{g} \mathrm{mL}^{-1}\right)$ or QD-RGD $\left(50 \mu \mathrm{g} \mathrm{mL}^{-1}\right)$ for $8 \mathrm{~h}$ under hypoxic conditions and subsequently treated with RT (6 Gy). At the end of the experiment, cells were fixed with $4 \%$ paraformaldehyde for $20 \mathrm{~min}$ and stained with crystal violet for 20 min following by washing with PBS. The CCK8 assay (Dojindo) was performed according to the manufacturer's protocol. 
a

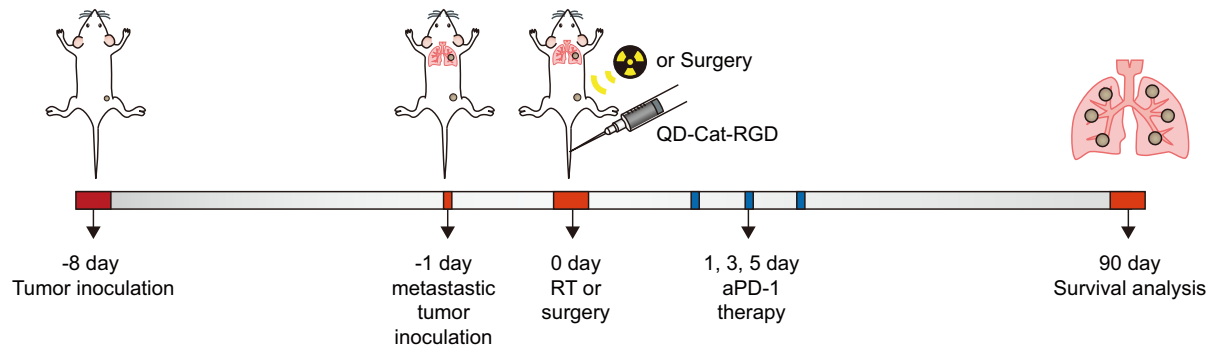

b

Day 10

Day 25

Day 35
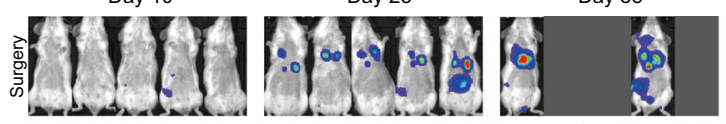

Day 45

Day 55
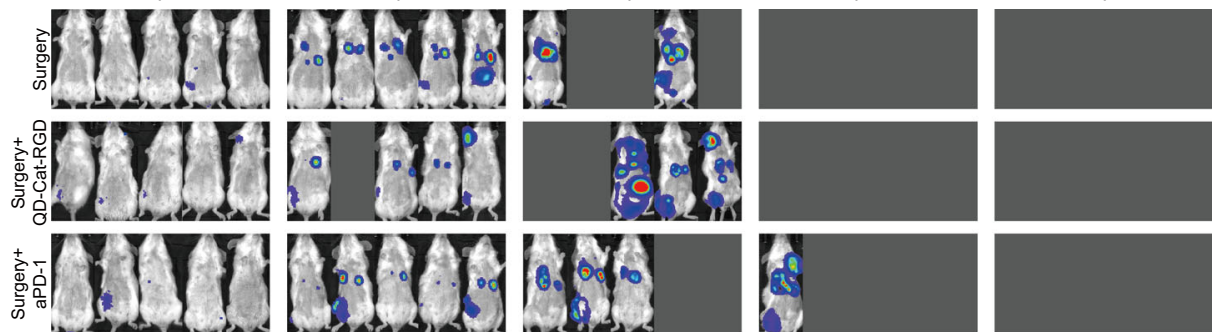

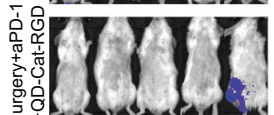
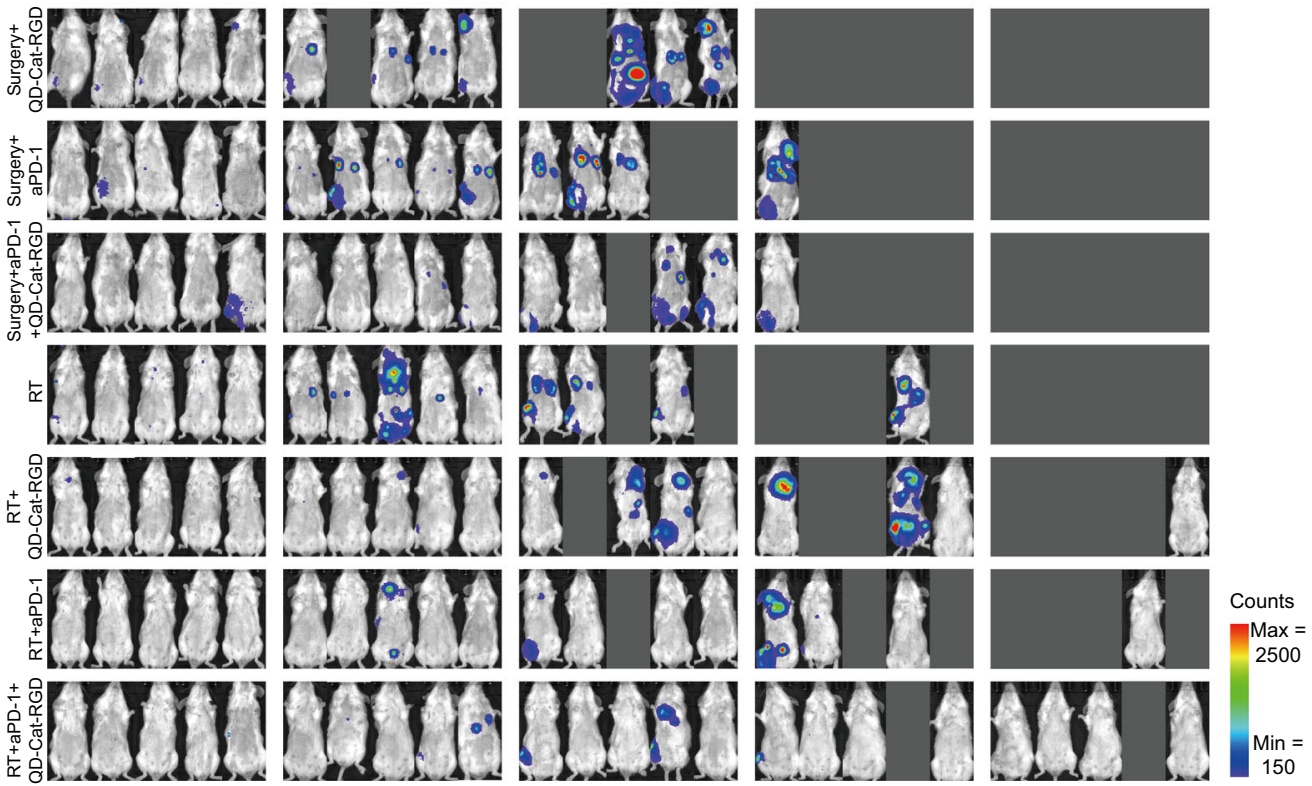

C
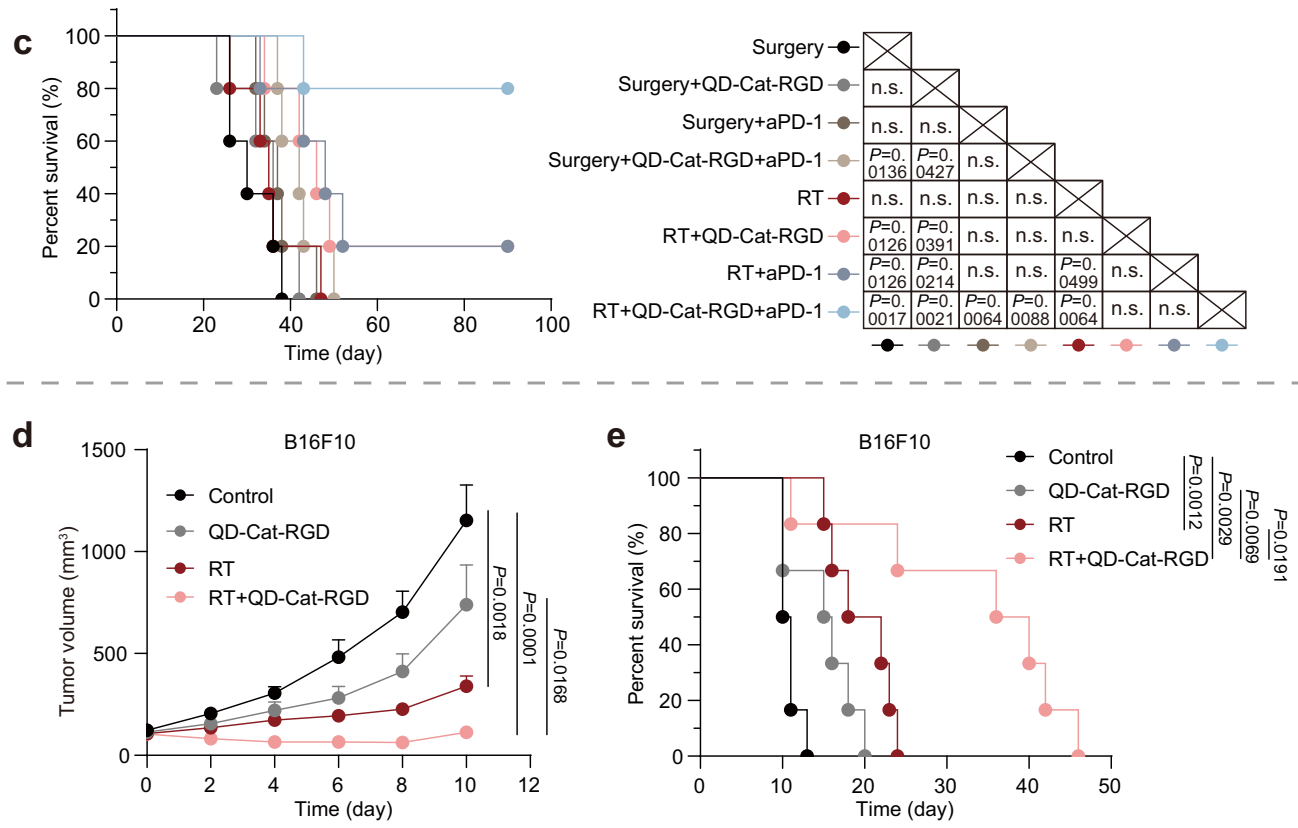

Cytokine and ATP detection. For the secretion levels of HMGB1 and ATP from cells in vitro, cells were first inoculated with QD-Cat-RGD $\left(50 \mu \mathrm{g} \mathrm{mL}^{-1}\right)$ or QDRGD $\left(50 \mu \mathrm{g} \mathrm{mL}^{-1}\right)$ for $8 \mathrm{~h}$ and then treated with RT (6 Gy). Culture medium samples were isolated from the cells with different treatments under hypoxic conditions $\left(37^{\circ} \mathrm{C}, 1 \% \mathrm{O}_{2}, 5 \% \mathrm{CO}_{2}\right)$ after $24 \mathrm{~h}$. HMGB1 was detected by a mouse HMGB1 ELISA kit (IBL-International, Cat ST51011). ATP was detected by an ATP assay kit (Beyotime, Cat S0026). Tumor tissues were collected from mice and then homogenized in PBS buffer. The protein concentrations were normalized by a BCA protein kit (Beyotime, Cat P0012S). The intratumoral levels of IFN- $\gamma$ (4A Biotech, Cat CME0003), TNF- $\alpha$ (4A Biotech, Cat CME0004), and IL-10 (4A Biotech, Cat CME0016) were measured with mouse ELISA kits according to the manufacturer's protocol. 
Fig. 7 QD-Cat-RGD-based RT for treating cancer in the 4T1 experimental early metastasis model and B16F10 melanoma model. a Schematic showing the experiment using QD-Cat-RGD-based RT to treat mice with the 4 T1 experimental model of metastasis at an early stage. BALB/c mice ( $n=5$ per group) were implanted subcutaneously with $7 \times 10^{5} 4 \mathrm{~T} 1$ mammary carcinoma cells on right hind flanks and after 7 days consequently implanted intravenously with $2 \times 10^{5} 4 \mathrm{~T} 1$ cells to mimic the early stage of metastasis in which cancer cells escaped from the primary tumor site into the circulation and seeded in distant organs especially the lung. When primary tumor volumes were $100 \mathrm{~mm}^{3}$, mice received different treatments. b Bioluminescence images tracking the spreading and growth of intravenously injected 4T1-Luc cells in BALB/c mice ( $n=5$ per group) treated with surgery, surgery + QD-Cat-RGD, surgery + aPD-1, surgery + QD-Cat-RGD + aPD-1, RT, RT + QD-Cat-RGD, RT + aPD-1 or RT + QD-Cat-RGD + aPD-1 on days 10, 25, 35, 45, and 55. c Survival analysis of $4 \mathrm{~T} 1$ lung metastatic mice ( $n=5$ per group) after the different treatments. $\mathbf{d}$ C57BL/ 6 mice $(n=6$ per group) were implanted subcutaneously with $3 \times 10^{5}$ B16F10 murine melanoma cells in the right hind flanks. When tumor volumes were $100 \mathrm{~mm}^{3}$, mice received PBS, QD-Cat-RGD, RT, or RT + QD-Cat-RGD treatment. Tumor growth curves of B16F10 tumors. Photographs of all tumor-bearing mice at day 8 are shown in Supplementary Fig. 17. e Survival analysis of mice ( $n=6$ per group) with B16F10 tumors treated with PBS (control), QD-Cat-RGD, RT or RT + QD-Cat-RGD. All data are presented as the mean \pm s.e.m. ( $n=5-6$ per group) and $n$ represents the number of independent animals. Statistical significance was calculated via oneway ANOVA with Tukey's multiple comparisons test (d) and log-rank (Mantel-Cox) test (c, e). n.s. not significant. Source data are provided as a Source Data file.

Animal experiments. To evaluate the therapeutic efficacy, female BALB/c and C57BL/6 mice (6-8 weeks) were purchased from Hubei Provincial Academy of Preventive Medicine. Ethical approval of this study was obtained from the Animal Ethics Committee of the School and Hospital of Stomatology of Wuhan University (approval number: S07920070E). All animal experimental procedures were performed in accordance with the Regulations for the Administration of Affairs Concerning Experimental Animals approved by the State Council of the People's Republic of China. All mice were housed under specific pathogen-free (SPF) conditions (temperature $\sim 22^{\circ} \mathrm{C}$, humidity $\sim 50 \%$ ) with a $12 / 12 \mathrm{~h}$ dark/light cycle. $4 \mathrm{~T} 1\left(7 \times 10^{5}\right)$ cells or B16F10 cells $\left(3 \times 10^{5}\right)$ resuspended in $100 \mu \mathrm{L}$ of RPMI medium were subcutaneously injected into the right hind flank of each BALB/c mouse or C57BL/6 mouse. After the tumor volume reached approximately $100 \mathrm{~mm}^{3}$, the mice were randomly divided into groups ( $n=5$ or 6 per group). The tumor-bearing mice were intravenously injected with a single dose of QD-CatRGD $\left(150 \mu \mathrm{L}, 2 \mathrm{mg} \mathrm{mL}^{-1}\right)$, QD-Cat $\left(150 \mu \mathrm{L}, 2 \mathrm{mg} \mathrm{mL}^{-1}\right)$, QD-RGD $(150 \mu \mathrm{L}$, $\left.2 \mathrm{mg} \mathrm{mL}^{-1}\right)$, or PBS $(150 \mu \mathrm{L})$. The location and range of RT with the help of NIRIIb imaging $2 \mathrm{~h}$ after injection (Supplementary Fig. 8a). Then, we used the lead shield to localize the range of RT determined by previous imaging (Supplementary Fig. 8b,c). A small animal irradiator (RS2000pro, Rad Source Technologies) was used in this experiment. Tumor volume was calculated as follows: width ${ }^{2} \times$ length $\times 0.5$. All mice in this study were euthanized under $\mathrm{CO}_{2}$ anesthesia if the volume of primary tumor reached a maximum allowable volume of $1,500 \mathrm{~mm}^{3}$ or if tumor burden compromised the animal welfare. For depletion of $\mathrm{CD} 8^{+} \mathrm{T}$ cells, the aCD8 antibodies (200 $\mu \mathrm{g}$ per mouse, Bioxcell, clone 53-6.7, Cat BE0004-1) were injected intraperitoneally on days $0,4,8$, and 12 .

To establish a distant metastasis model, female BALB/c mice (6-8 weeks) were purchased from Hubei Provincial Academy of Preventive Medicine. For the primary tumor inoculation, 4 T1 cells $\left(7 \times 10^{5}\right)$ suspended in $100 \mu \mathrm{L}$ RPMI medium were subcutaneously injected into the right hind flank of each female BALB/c mouse. Two days after the first tumor inoculation, $4 \mathrm{~T} 1$ cells $\left(7 \times 10^{5}\right)$ were subcutaneously injected into the left hind flank of each female BALB/c mouse as a distant tumor mimic. Eight days after the first tumor inoculation, the tumorbearing mice were treated with PBS (control group), QD-Cat-RGD, aPD-1, QDCat-RGD + aPD-1, RT (12 Gy), RT + QD-Cat-RGD, RT + aPD-1, and RT + QDCat-RGD + aPD-1. QD-Cat-RGD was injected into mice via the tail vein. The aPD-1 antibodies (200 $\mu \mathrm{g}$ per mouse, Bioxcell, clone RMP1-14, Cat BE0146) were injected intraperitoneally on days 1,3 , and 5 .

To establish the experimental model of metastasis at early stage, 7 days after primary tumor injection, luciferase-transgenic $4 \mathrm{~T} 1(4 \mathrm{~T} 1-\mathrm{Luc})$ cells $\left(2 \times 10^{5}\right)$ were administered intravenously via tail vein infusion into each BALB/c mouse. Eight days after the first tumor inoculation, $4 \mathrm{~T} 1$ tumor-bearing mice were treated with surgery, surgery + QD-Cat-RGD, surgery + aPD-1, surgery + QD-Cat-

$\mathrm{RGD}+\mathrm{aPD}-1, \mathrm{RT}, \mathrm{RT}+\mathrm{QD}-\mathrm{Cat}-\mathrm{RGD}, \mathrm{RT}+\mathrm{aPD}-1$ or RT + QD-CatRGD + aPD-1. The aPD-1 antibodies were injected intraperitoneally on days 1,3 , and 5. Metastatic cancer was monitored by the IVIS Spectrum Imaging System (PerkinElmer) and analyzed by Living Image software (PerkinElmer). Before the mice were imaged, $\mathrm{D}$-luciferin $\left(150 \mathrm{mg} \mathrm{kg}^{-1}\right)$ was intraperitoneally injected into the mice.

Western blot analysis. Tissue harvested from treated mice was lysed with RIPA buffer (Beyotime). The protein concentrations were normalized by a BCA protein kit (Beyotime, Cat P0012S). Western blot analysis was performed as previously described $^{64}$. Briefly, the proteins were separated by SDS-PAGE using wet electroblotting systems (Bio-Rad) and transferred to PVDF membranes (Roche). Membranes were blocked in nonfat milk (5\%) for $1 \mathrm{~h}$ and incubated with primary antibodies overnight at $4{ }^{\circ} \mathrm{C}$. Antibodies against the following proteins were used for the western blot assay: GAPDH (1:5,000, Proteintech, Cat HRP-60004) and HIF-1 $\alpha$ (1:500, Novus Biologicals, Cat NB-100-105). Signal detection was performed by using an ECL kit (Advansta). The signals were collected and analyzed using an Odyssey system (Li-Cor Biosciences) and Image Studio software v. 5.2 (LiCor Biosciences).

IHC and histological analysis. H\&E staining and IHC staining were performed as previously described ${ }^{65}$. Briefly, after deparaffinization and rehydration, $4 \mu \mathrm{m}$ paraffin-embedded tumor tissue sections were counterstained with H\&E (ZSGB$\mathrm{BIO})$ and then dehydrated with graded alcohol and clearing in xylene. For IHC, after deparaffinization and rehydration, $4 \mu \mathrm{m}$ paraffin-embedded tumor tissue sections were retrieved by citrate acid solution $(\mathrm{pH} 6.0)$ for $5 \mathrm{~min}$ at high pressure. The slides were subsequently incubated in $3 \%$ hydrogen peroxide and in goat serum. Primary antibodies against CD3 $\varepsilon$ (1:200, Cell Signaling Technology, Cat 99940), CD8a (1:400, Cell Signaling Technology, Cat 98941), Foxp3 (1:400, Cell Signaling Technology, Cat 12653), granzyme B (1:200, Cell Signaling Technology, Cat 44153), and HIF-1a (1:150, Novus, Cat NB100-105) were used. HRPconjugated secondary antibodies (undiluted, anti-rabbit/anti-mouse, Cell Signaling Technology, Cat $8114 / 8125$ ) were then used. The signals were detected by DAB substrate kit (Cell Signaling Technology). Slides were scanned using an Aperio ScanScope CS scanner (Leica). CaseViewer v. 2.4 (3DHISTECH) was used for creating slide presentations. Aperio ImageScope software v. 12.3.2 (Leica) was used for histoscore analysis of these detection indicators. The histoscore was normalized to $0-300$.

Immunofluorescence. Immunofluorescence staining was performed as previously described $^{64}$. Primary antibodies against anti-phospho-histone H2AX (Ser139/ Tyr142) (1:100, Cell Signaling Technology, Cat 5438), calreticulin (1:400, Cell Signaling Technology, Cat 12238), and HMGB1 (1:400, Abcam, Cat ab18256) were used. Fluorescence-conjugated secondary antibodies (1:500, anti-rabbit, Dylight 488/594, Abbkine, Cat 23220/23430) and anti-fade fluorescence mounting medium with DAPI (ZSGB-BIO) were then used. The slides were examined with an FV1000 confocal microscope (Olympus) using FV10-ASW software v. 4.0 (Olympus). The fluorescence signals were analyzed using Image-Pro Plus software v. 6.0 (Media Cybernetics).

Flow cytometric analysis and FACS. For detection of calreticulin expression on the cell surface, an anti-calreticulin antibody (1:100, Cell Signaling Technology, Alexa Fluor 488, Cat 62304) was used to stain the cells after different treatments. For control, rabbit IgG isotype control (1:100, Cell Signaling Technology, Alexa Fluor 488, Cat 2975) was used. For the annexin V/PI assay, cells were digested by EDTA-free trypsin and washed with PBS buffer followed by detection with Annexin V apoptosis detection kit (eBioscience). The stained cells were run on a flow cytometer (Beckman) using the CytExpert software v. 2.3 (Beckman) and then analyzed by FlowJo software (v. 10, TreeStar).

For lymphocyte analysis in vivo, tumors and TDLNs were collected from mice and made into cell suspensions by gentleMACS ${ }^{\mathrm{mt}}$ dissociator and digestive enzyme (Miltenyi Biotec) according to the manufacturer's instructions. Live or dead cells were separated by Fixable Viability Dye (eBioscience, eFluor 780 Cat 65-0865-14). Cells were stained with fluorescence-labeled anti-CD45 (1:500, eBioscience, perCPCyanine5.5, clone 30-F11, Cat 45-0451-82), anti-CD3e (1:500, eBioscience, FITC, clone 145-2C11, Cat 11-0031-82), anti-CD4 (1:500, eBioscience, eFluor 450, clone RM4-5, Cat 48-0042-82), anti-CD8a (1:500, Biolegend, APC, clone 53-6.7, Cat 100712), anti-CD8a (1:500, Biolegend, Alexa Fluor 594, clone 53-6.7, Cat 100758), anti-CD11b (1:500, Biolegend, FITC, clone M1/70, Cat 101206), anti-F4/80 (1:500, eBioscience, PE-Cyanine7, clone BM8, Cat 25-4801-82), anti-CD80 (1:300, eBioscience, APC, clone 16-10A1, Cat 17-0801-82), anti-CD206 (1:200, Biolegend, $\mathrm{PE}$, clone C068C2, Cat 141705), anti-CD11c (1:500, eBioscience, FITC, clone N418, Cat 11-0114-82), anti-CD80 (1:300, eBioscience, PE, clone 16-10A1, Cat 12-080182), anti-CD86 (1:300, eBioscience, APC, clone GL1, Cat 17-0862-82), anti-MHCII (1:300, eBioscience, PE-Cyanine7, clone M5/114.15.2, Cat 25-5321-82), antiCD25 (1:200, eBioscience, APC, clone PC61.5, Cat 17-0251-82), anti-Foxp3 (1:100, 
eBioscience, PE, clone FJK-16s, Cat 12-5773-82), anti-CD44 (1:500, eBioscience, PE, clone IM7, Cat 12-0441-82), and anti-CD62L (1:200, eBioscience, APC, clone MEL-14, Cat 17-0621-82) antibodies following the manufacturers' instructions. The stained cells run on a flow cytometer (Beckman) using the CytExpert software v. 2.3 (Beckman). The gating strategy used for the study is shown in Supplementary Fig. 9. The positive gate of $\mathrm{CD} 206^{\text {high }}$ or $\mathrm{CD} 80^{\text {high }} \mathrm{TAMs}$ were determined by fluorescence-minus-one (FMO) control ${ }^{66}$. The data acquired flow cytometer was analyzed by the FlowJo software (v. 10, TreeStar).

To isolate the live $\mathrm{CD} 45^{-}$cells from tumor tissue, single cell suspension of tumor tissue was stained with the Fixable Viability Dye (eBioscience, eFluor 780, Cat 65-0865-14) and anti-CD45 (1:500, eBioscience, perCP-Cyanine5.5, clone 30F11, Cat 45-0451-82) antibody. Live $\mathrm{CD}^{-} 5^{-}$cells (the sorting gating strategy is shown in Supplementary Fig. 3a) were sorted on a MoFlo XDP cell sorter (Beckman) in PBS with 2\% FBS

Statistical analysis. Data are represented as the mean \pm standard error of the mean (s.e.m.) or standard deviation (s.d.) as indicated in the figure legends. Oneway ANOVA with Tukey's multiple comparisons was used for multiple comparisons when more than two groups were compared, and two-tailed Student's $t$-test was used for two-group comparisons. Survival benefit was determined using a logrank (Mantel-Cox) test. All statistical tests were performed using GraphPad Prism software v. 8.0 (GraphPad Software) and Excel 2016 software (Microsoft). In all types of statistical analysis values of $P<0.05$ were considered significant.

Reporting summary. Further information on research design is available in the Nature Research Reporting Summary linked to this article.

\section{Data availability}

The data generated in this study are available within the Article, Supplementary Information or Source Data file. Source data are provided with this paper.

Received: 6 January 2021; Accepted: 24 November 2021; Published online: 09 December 2021

\section{References}

1. Siegel, R. L., Miller, K. D. \& Jemal, A. Cancer statistics, 2020. CA Cancer J. Clin. 70, 7-30 (2020).

2. Yap, M. L., Zubizarreta, E., Bray, F., Ferlay, J. \& Barton, M. Global Access to Radiotherapy Services: Have We Made Progress During the Past Decade? J. Glob. Oncol. 2, 207-215 (2016).

3. De Ruysscher, D. et al. Radiotherapy toxicity. Nat. Rev. Dis. Prim. 5, 13 (2019).

4. Vaupel, P., Kallinowski, F. \& Okunieff, P. Blood flow, oxygen and nutrient supply, and metabolic microenvironment of human tumors: a review. Cancer Res. 49, 6449-6465 (1989).

5. Wouters, B. G. \& Brown, J. M. Cells at intermediate oxygen levels can be more important than the "hypoxic fraction" in determining tumor response to fractionated radiotherapy. Radiat. Res. 147, 541-550 (1997).

6. Fisher, B. et al. Five-year results of a randomized clinical trial comparing total mastectomy and segmental mastectomy with or without radiation in the treatment of breast cancer. N. Engl. J. Med. 312, 665-673 (1985).

7. Lambrecht, M., Dirix, P., Van den Bogaert, W. \& Nuyts, S. Incidence of isolated regional recurrence after definitive (chemo-) radiotherapy for head and neck squamous cell carcinoma. Radiother. Oncol. 93, 498-502 (2009).

8. Weichselbaum, R. R., Liang, H., Deng, L. \& Fu, Y. X. Radiotherapy and immunotherapy: a beneficial liaison? Nat. Rev. Clin. Oncol. 14, 365-379 (2017).

9. Galluzzi, L., Buque, A., Kepp, O., Zitvogel, L. \& Kroemer, G. Immunogenic cell death in cancer and infectious disease. Nat. Rev. Immunol. 17, 97-111 (2017).

10. Rodriguez-Ruiz, M. E., Vitale, I., Harrington, K. J., Melero, I. \& Galluzzi, L. Immunological impact of cell death signaling driven by radiation on the tumor microenvironment. Nat. Immunol. 21, 120-134 (2020).

11. Ngwa, W. et al. Using immunotherapy to boost the abscopal effect. Nat. Rev. Cancer 18, 313-322 (2018).

12. Kang, J., Demaria, S. \& Formenti, S. Current clinical trials testing the combination of immunotherapy with radiotherapy. J. Immunother. Cancer 4 , 51 (2016).

13. Overgaard, J. Hypoxic modification of radiotherapy in squamous cell carcinoma of the head and neck-a systematic review and meta-analysis. Radiother. Oncol. 100, 22-32 (2011).

14. Yoshimura, M., Itasaka, S., Harada, H. \& Hiraoka, M. Microenvironment and radiation therapy. Biomed. Res. Int. 2013, 685308 (2013).

15. Moeller, B. J., Cao, Y. T., Li, C. Y. \& Dewhirst, M. W. Radiation activates HIF1 to regulate vascular radiosensitivity in tumors: role of reoxygenation, free radicals, and stress granules. Cancer Cell 5, 429-441 (2004).
16. Doedens, A. L. et al. Macrophage expression of hypoxia-inducible factor-1 alpha suppresses $\mathrm{T}$-cell function and promotes tumor progression. Cancer Res. 70, 7465-7475 (2010)

17. Laoui, D. et al. Tumor hypoxia does not drive differentiation of tumorassociated macrophages but rather fine-tunes the M2-like macrophage population. Cancer Res. 74, 24-30 (2014).

18. Rolny, C. et al. HRG inhibits tumor growth and metastasis by inducing macrophage polarization and vessel normalization through downregulation of PlGF. Cancer Cell 19, 31-44 (2011).

19. Facciabene, A. et al. Tumour hypoxia promotes tolerance and angiogenesis via CCL28 and T(reg) cells. Nature 475, 226-230 (2011).

20. Song, G., Cheng, L., Chao, Y., Yang, K. \& Liu, Z. Emerging nanotechnology and advanced materials for cancer radiation therapy. Adv. Mater. 29, 1700996 (2017).

21. Fan, W. et al. Breaking the depth dependence by nanotechnology-enhanced X-ray-excited deep cancer theranostics. Adv. Mater. 31, 1806381 (2019).

22. Du, J. et al. Design of TPGS-functionalized Cu3BiS3 nanocrystals with strong absorption in the second near-infrared window for radiation therapy enhancement. Nanoscale 9, 8229-8239 (2017).

23. Du, J. et al. Poly(vinylpyrollidone)- and selenocysteine-modified Bi2Se3 nanoparticles enhance radiotherapy efficacy in tumors and promote radioprotection in normal tissues. Adv. Mater. 29, 1701268 (2017).

24. Yang, Y. et al. NIR-II driven plasmon-enhanced catalysis for a timely supply of oxygen to overcome hypoxia-induced radiotherapy tolerance. Angew. Chem. Int. Ed. 58, 15069-15075 (2019).

25. Yu, C. Y. Y. et al. Mitochondrion-anchoring photosensitizer with aggregationinduced emission characteristics synergistically boosts the radiosensitivity of cancer cells to ionizing radiation. Adv. Mater. 29, 1606167 (2017).

26. Chen, Q. et al. Nanoparticle-enhanced radiotherapy to trigger robust cancer immunotherapy. Adv. Mater. 31, 1802228 (2019).

27. Prasad, P. et al. Multifunctional albumin- $\mathrm{MnO}_{2}$ nanoparticles modulate solid tumor microenvironment by attenuating hypoxia, acidosis, vascular endothelial growth factor and enhance radiation response. ACS Nano 8, 3202-3212 (2014).

28. Wan, H., Du, H., Wang, F. \& Dai, H. Molecular imaging in the second nearinfrared window. Adv. Funct. Mater. 29, 1900566 (2019).

29. Zhong, Y. et al. In vivo molecular imaging for immunotherapy using ultrabright near-infrared-IIb rare-earth nanoparticles. Nat. Biotechnol. 37, 1322-1331 (2019).

30. Zhang, M. et al. Bright quantum dots emitting at approximately $1,600 \mathrm{~nm}$ in the NIR-IIb window for deep tissue fluorescence imaging. Proc. Natl Acad. Sci. USA 115, 6590-6595 (2018).

31. Huang, B. et al. Near-infrared IIb emitting nanoprobe for high-resolution realtime imaging-guided photothermal therapy triggering enhanced anti-tumor immunity. ACS Appl. Bio Mater. 3, 1636-1645 (2020).

32. Hermanson, G. T. Bioconjugate Techniques (Academic Press, 2008).

33. Boyer, R. F. Modern Experimental Biochemistry (Prentice Hall PTR, 2000).

34. Wang, L. et al. Construction of nucleus-targeting iridium nanocrystals for photonic hyperthermia-synergized cancer radiotherapy. Small 15, e1903254 (2019).

35. Guo, Z. et al. Targeting efficiency of RGD-modified nanocarriers with different ligand intervals in response to integrin alphavbeta3 clustering. Biomaterials 35, 6106-6117 (2014).

36. Xie, J. et al. Emerging strategies of nanomaterial-mediated tumor radiosensitization. Adv. Mater. 31, 1802244 (2019).

37. Wilhelm, S. et al. Analysis of nanoparticle delivery to tumours. Nat. Rev. Mater. 1, 16014 (2016).

38. Rogakou, E. P., Pilch, D. R., Orr, A. H., Ivanova, V. S. \& Bonner, W. M. DNA double-stranded breaks induce histone H2AX phosphorylation on serine 139. J. Biol. Chem. 273, 5858-5868 (1998).

39. Wang, X. et al. Enhanced generation of non-oxygen dependent free radicals by Schottky-type heterostructures of Au-Bi2S3 nanoparticles via X-ray-induced catalytic reaction for radiosensitization. ACS Nano 13, 5947-5958 (2019).

40. Barker, H. E., Paget, J. T., Khan, A. A. \& Harrington, K. J. The tumour microenvironment after radiotherapy: mechanisms of resistance and recurrence. Nat. Rev. Cancer 15, 409-425 (2015).

41. Kachikwu, E. L. et al. Radiation enhances regulatory T cell representation. Int. J. Radiat. Oncol. Biol. Phys. 81, 1128-1135 (2011).

42. Wculek, S. K. et al. Dendritic cells in cancer immunology and immunotherapy. Nat. Rev. Immunol. 20, 7-24 (2020).

43. van der Leun, A. M., Thommen, D. S. \& Schumacher, T. N. CD8(+) T cell states in human cancer: insights from single-cell analysis. Nat. Rev. Cancer 20, 218-232 (2020)

44. Liu, C. et al. Neuropilin-1 is a T cell memory checkpoint limiting long-term antitumor immunity. Nat. Immunol. 21, 1010-1021 (2020).

45. Reading, J. L. et al. The function and dysfunction of memory CD8(+) T cells in tumor immunity. Immunol. Rev. 283, 194-212 (2018).

46. DePeaux, K. \& Delgoffe, G. M. Metabolic barriers to cancer immunotherapy. Nat. Rev. Immunol. 21, 785-797 (2021). 
47. Dong, Z., Yang, Z., Hao, Y. \& Feng, L. Fabrication of $\mathrm{H}_{2} \mathrm{O}_{2}$-driven nanoreactors for innovative cancer treatments. Nanoscale 11, 16164-16186 (2019).

48. Golden, E. B., Demaria, S., Schiff, P. B., Chachoua, A. \& Formenti, S. C. An abscopal response to radiation and ipilimumab in a patient with metastatic non-small cell lung cancer. Cancer Immunol. Res. 1, 365-372 (2013).

49. Demaria, S. et al. Ionizing radiation inhibition of distant untreated tumors (abscopal effect) is immune mediated. Int. J. Radiat. Oncol. Biol. Phys. 58, 862-870 (2004).

50. Togashi, Y., Shitara, K. \& Nishikawa, H. Regulatory T cells in cancer immunosuppression-implications for anticancer therapy. Nat. Rev. Clin. Oncol. 16, 356-371 (2019).

51. Mantovani, A., Marchesi, F., Malesci, A., Laghi, L. \& Allavena, P. Tumourassociated macrophages as treatment targets in oncology. Nat. Rev. Clin. Oncol. 14, 399-416 (2017).

52. Seyfried, T. N. \& Huysentruyt, L. C. On the origin of cancer metastasis. Crit. Rev. Oncog. 18, 43-73 (2013).

53. Gengenbacher, N., Singhal, M. \& Augustin, H. G. Preclinical mouse solid tumour models: status quo, challenges and perspectives. Nat. Rev. Cancer 17, 751-765 (2017).

54. Li, P. et al. Lung mesenchymal cells elicit lipid storage in neutrophils that fuel breast cancer lung metastasis. Nat. Immunol. 21, 1444-1455 (2020).

55. Headley, M. B. et al. Visualization of immediate immune responses to pioneer metastatic cells in the lung. Nature 531, 513-517 (2016).

56. Min, Y. et al. Antigen-capturing nanoparticles improve the abscopal effect and cancer immunotherapy. Nat. Nanotechnol. 12, 877-882 (2017).

57. Gregory, J. V. et al. Systemic brain tumor delivery of synthetic protein nanoparticles for glioblastoma therapy. Nat. Commun. 11, 5687 (2020).

58. $\mathrm{Ni}, \mathrm{K}$. et al. Nanoscale metal-organic frameworks enhance radiotherapy to potentiate checkpoint blockade immunotherapy. Nat. Commun. 9, 2351 (2018).

59. Irvine, D. J. \& Dane, E. L. Enhancing cancer immunotherapy with nanomedicine. Nat. Rev. Immunol. 20, 321-334 (2020)

60. Zhang, M.-X., Huang, B.-H., Sun, X.-Y. \& Pang, D.-W. Clickable gold nanoparticles as the building block of nanobioprobes. Langmuir $\mathbf{2 6}$ 10171-10176 (2010).

61. Yu, G. T. et al. Molecular targeting nanoprobes with non-overlap emission in the second near-infrared window for in vivo two-color colocalization of immune cells. ACS Nano 13, 12830-12839 (2019).

62. Zhang, Y., Huo, M., Zhou, J. \& Xie, S. PKSolver: an add-in program for pharmacokinetic and pharmacodynamic data analysis in Microsoft Excel. Comput. Methods Prog. Biomed. 99, 306-314 (2010).

63. Franken, N. A., Rodermond, H. M., Stap, J., Haveman, J. \& van Bree, C. Clonogenic assay of cells in vitro. Nat. Protoc. 1, 2315-2319 (2006)

64. Chen, L. et al. Targeting CMTM6 suppresses stem cell-like properties and enhances antitumor immunity in head and neck squamous cell carcinoma. Cancer Immunol. Res. 8, 179-191 (2020).

65. Li, H. et al. High expression of GPNMB predicts poor prognosis in head and neck squamous cell carcinoma. Histol. Histopathol. 34, 803-810 (2019).

66. Perfetto, S. P., Chattopadhyay, P. K. \& Roederer, M. Seventeen-colour flow cytometry: unravelling the immune system. Nat. Rev. Immunol. 4, 648-655 (2004).

\section{Acknowledgements}

This work is supported by the National Key R\&D Program of China (2020YFA0908800, 2017YFSF090107) and the National Natural Science Foundation of China grant to Z.-J.S. (82072996 and 81874131), M.Z. (21974104), and R.C. (22174105). We thank Shuyan Liang and Zhixin Qiu from Wuhan Biobank Co., Ltd (Wuhan, China) for their excellent technical assistance on flow cytometry and FACS.

\section{Author contributions}

Z.-J.S., M.Z., and R.C. conceived and designed the experiments. H.L., M.W., B.H., S.-W.Z., J.-J.Z., and D.-R.C. performed the experiments. H.L., M.W., and B.H. analyzed the data. H.L., M.W., Z.-J.S., M.Z., and R.C. wrote the manuscript. All authors have given approval to the final version of the manuscript.

\section{Competing interests}

The authors declare no competing interests.

\section{Additional information}

Supplementary information The online version contains supplementary material available at https://doi.org/10.1038/s41467-021-27485-0.

Correspondence and requests for materials should be addressed to Ran Cui, Mingxi Zhang or Zhi-Jun Sun.

Peer review information Nature Communications thanks Zhuang Liu and the other, anonymous, reviewer(s) for their contribution to the peer review of this work. Peer reviewer reports are available.

Reprints and permission information is available at http://www.nature.com/reprints

Publisher's note Springer Nature remains neutral with regard to jurisdictional claims in published maps and institutional affiliations.

cc) (i) Open Access This article is licensed under a Creative Common Attribution 4.0 International License, which permits use, sharing, adaptation, distribution and reproduction in any medium or format, as long as you give appropriate credit to the original author(s) and the source, provide a link to the Creative Commons license, and indicate if changes were made. The images or other third party material in this article are included in the article's Creative Commons license, unless indicated otherwise in a credit line to the material. If material is not included in the article's Creative Commons license and your intended use is not permitted by statutory regulation or exceeds the permitted use, you will need to obtain permission directly from the copyright holder. To view a copy of this license, visit http://creativecommons.org/ licenses/by/4.0/.

(C) The Author(s) 2021 